\title{
CP violation in multibody decays of beauty baryons
}

\author{
Gauthier Durieux \\ $D E S Y$, \\ Notkestrasse 85, D-22607 Hamburg, Germany \\ E-mail: gauthier.durieux@desy.de
}

ABSTRACT: Beauty baryons are being observed in large numbers in the LHCb detector. The rich kinematic distributions of their multibody decays are therefore becoming accessible and provide us with new opportunities to search for $\mathrm{CP}$ violation. We analyse the angular distributions of some three- and four-body decays of spin- $1 / 2$ baryons using the Jacob-Wick helicity formalism. The asymmetries that provide access to small differences of CP-odd phases between decay amplitudes of identical CP-even phases are notably discussed. The understanding gained on processes featuring specific resonant intermediate states allows us to establish which asymmetries are relevant for what purpose. It is for instance shown that some CP-odd angular asymmetries measured by the LHCb collaboration in the $\Lambda_{b} \rightarrow$ $\Lambda \varphi \rightarrow p \pi K^{+} K^{-}$decay are expected to vanish identically.

KeYwORDS: CP violation, Heavy Quark Physics

ARXiv EPrint: 1608.03288 


\section{Contents}

1 Introduction $\quad 1$

2 Angular distributions $\quad 4$

$\begin{array}{lll}3 & \text { Discrete symmetry properties } & 7\end{array}$

4 Asymmetries 14

4.1 T-odd angular asymmetries $\quad 14$

$\begin{array}{lll}4.2 & \hat{T} \text {-even angular asymmetries } & 16\end{array}$

$\begin{array}{lll}5 & \text { Summary } & 17\end{array}$

$\begin{array}{lr}\text { A Extended angular distributions } & 18\end{array}$

\section{Introduction}

Despite of production rates somewhat smaller than that of mesons, beauty baryons are now being observed in significant numbers in the LHCb detector. They have therefore started to offer complementary means to test the standard model. The search for new sources of $\mathrm{CP}$ violation is an especially relevant direction in which they could provide new opportunities. Incidentally, a first hint of $\mathrm{CP}$ violation could just have been observed in the $\Lambda_{b} \rightarrow p \pi^{-} \pi^{+} \pi^{-}$channel [1]. Using angular momentum conservation through the JacobWick helicity formalism, we aim at determining what angular asymmetries can be expected in specific beauty baryon decays as well as how they relate to the underlying dynamics and its discrete symmetry properties. By discussing the case of spin- $1 / 2$ baryon decays, this paper extends ref. [2] that focused on the case of spin-0 particles. Most of our results also apply to the decay of any spin- $1 / 2$ state.

A violation of $\mathrm{CP}$, sourced in the standard model or beyond, manifests itself through relative CP-odd phases — also called weak phases — between decay amplitudes. They can be accessed through interferences in which CP-even — or strong — phases originating from the absorptive parts of amplitudes can also appear. The most common interferences take the following form:

$$
\begin{aligned}
\operatorname{Re}\left\{A_{1}^{*} A_{2}\right\} & =\left|A_{1}^{*} A_{2}\right| \operatorname{Re}\left\{e^{i \Delta \delta_{12}+i \Delta \varphi_{12}}\right\} \\
& =\left|A_{1}^{*} A_{2}\right|\left(\cos \Delta \delta_{12} \cos \Delta \varphi_{12}-\sin \Delta \delta_{12} \sin \Delta \varphi_{12}\right)
\end{aligned}
$$

where $\Delta \varphi$ and $\Delta \delta$ respectively denote CP-odd and CP-even phase differences. The second $\mathrm{CP}$-odd term can be extracted by combining $\mathrm{CP}$-conjugate processes, through rate 
asymmetries notably. It provides sensitivity to small differences of weak phases, a sensitivity which is however conditioned on the presence of relative strong phases. Some other interferences take the

$$
\operatorname{Im}\left\{A_{1}^{*} A_{2}\right\}=\left|A_{1}^{*} A_{2}\right|\left(\sin \Delta \delta_{12} \cos \Delta \varphi_{12}+\cos \Delta \delta_{12} \sin \Delta \varphi_{12}\right)
$$

form. The second CP-odd term extracted by combining CP-conjugate processes is again sensitive to small weak phase differences, but does not vanish in the absence of relative strong phases. Studying this second type of interferences is therefore particularly relevant in cases where small strong phases are expected. Measuring both types of interferences can also lead to a better understanding of strong phases which are difficult to compute when they result from nonperturbative dynamics.

Beside rate asymmetries already mentioned, differential distributions can serve to access various interference terms. Exploiting the distributions of decay products instead of decay rates can also be advantageous when the production cross sections of CP-conjugate particles differ - as they generally do in $p p$ collisions - and production rate asymmetries are not precisely known. It is useful to define motion reversal $\hat{\mathrm{T}}$ (often called naive time reversal), a transformation that reverts momentum and spin three-vectors. Indeed, the motion reversal properties of differential distributions determine which type of amplitude interferences they give access to: $\hat{\mathrm{T}}$-even observables provide access to the $\operatorname{Re}\left\{A_{i}^{*} A_{j}\right\}$ interferences, $\hat{\mathrm{T}}$-odd observables to the $\operatorname{Im}\left\{A_{i}^{*} A_{j}\right\}$ ones. Let us focus somewhat on the $\hat{\mathrm{T}}$-odd observables which thus yield sensitivity to small differences of CP-odd phases between amplitudes having small or vanishing relative CP-even phases. In a Lorentz-invariant form, $\hat{\mathrm{T}}$-odd variables only appear proportional to a completely antisymmetric $\epsilon_{\mu \nu \rho \sigma}$ contraction of four independent four-vectors. In processes involving only spinless external states, they can thus only be constructed when at least five external particles are involved, like in fourbody decays. In processes involving spinning particles, $\hat{T}$-odd variables can in principle also be constructed through the antisymmetric contraction of both momentum and spin four-vectors. They constitute qualitatively different observables. Unlike momenta, the spin vectors of stable particles are however practically unmeasurable in the context we are interested in. So we will refrain from considering as observables the $\epsilon_{\mu \nu \rho \sigma}$ contractions in which they appear (that give rise to triple products like $\boldsymbol{s} \cdot\left(\boldsymbol{p}_{i} \times \boldsymbol{p}_{j}\right)$ in a specific frame). Only angular distributions that derive from measured final-state momenta will be awarded that status. Final-state spins will be altogether disregarded and summed over. The polarisation of the decaying particle can however be considered as resulting from the production process since it is determined by production amplitudes. In the decay of spinning particles, the angular distributions of decay products can then be viewed as providing access to combinations of production and decay amplitudes. This is to be contrasted with the decays of spinless particles where they provide direct access to decay amplitudes.

From this more practical point of view, here is how spinning particles offer new opportunities to search for small differences of CP-odd phases between decay amplitudes that have identical - potentially vanishing - CP-even phases. As a matter of fact, $\hat{\mathrm{T}}$-even angular distributions still provide access to small CP-odd phase differences only in the 
presence of relative $\mathrm{CP}$-even phases. The latter can however appear in the production amplitudes, as angular distributions now give access to an entwined combination of production and decay amplitudes. Such strong phases in production amplitudes would manifest themselves as a nonvanishing $\hat{\mathrm{T}}$-odd polarisation component, which we will denote $P_{z}$. As a result, certain imaginary parts of decay amplitude interferences become accessible through $\hat{\mathrm{T}}$-even angular distributions, in terms proportional to this $\hat{\mathrm{T}}$-odd polarisation component of the decaying particle. In particular, there are not enough independent external-particle four-momenta in three-body decays to form an antisymmetric $\epsilon_{\mu \nu \rho \sigma}$ contraction. One must necessarily rely on at least one spin four-vector to form a $\hat{\mathrm{T}}$-odd variable. As will be illustrated below with final-state spins summed over, the imaginary parts of decay amplitude interferences then only appear in terms proportional to the decaying particle polarisation. A positive signal of $\mathrm{CP}$ violation in one of the corresponding asymmetries could thus be sourced either in decay amplitudes or in production ones, leading, in the latter case, to a mismatch between the polarisation of the initial particle and minus the polarisation of its antiparticle. Such an effect is not expected to be sizeable when the strong interaction which conserves $\mathrm{CP}$ dominates the production process. Without assuming it is altogether absent, one would have to rely on a comparison between the expected and measured patterns of asymmetries to discriminate between these two possibilities. The patterns expected for decays through specific resonant intermediate states are presented below.

On the other hand, all the $\hat{\mathrm{T}}$-odd angular distributions can no longer serve to isolate small differences of CP-odd phases between decay amplitudes of identical CP-even phases. The $\hat{\mathrm{T}}$-odd angular distributions that appear proportional to $P_{z}$ no longer give access to imaginary parts of decay amplitude interferences. Sensitivity to hypothetical CP-odd phase differences through these terms then actually relies on the presence of nonvanishing CP-even phase differences between the corresponding decay amplitudes. A systematic and blind construction of $\hat{\mathrm{T}}$-odd-CP-odd asymmetries, as performed in ref. [2] for the decay of spinless particles, is nevertheless possible. We stress this procedure can still be utilized experimentally to cover the unexpected or in situations where complicated patterns of interferences are not described precisely enough. One would however need to rely on specific results such as the ones presented here for selected resonance structures to establish whether a given $\hat{\mathrm{T}}$-odd angular asymmetry yields sensitivity to CP-odd phase differences between production or decay amplitudes.

Aside from $\mathrm{CP}$ violation, one can in principle measure all prescribed independent contributions to the angular distributions and thereby gain further understanding about the process under scrutiny. Our tables establish the necessary link between kinematic distributions and the dynamics encoded in amplitudes. The precision achieved will obviously depend on the collected statistics, but note the determination of each asymmetry or moment exploits the statistical power of the full data sample. This is to be contrasted with a fit in which additional free parameters worsen the precision to which all of them can be determined (see e.g. ref. [3]). 

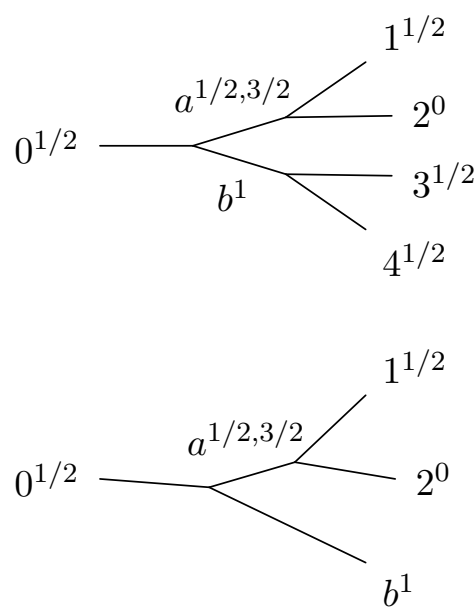
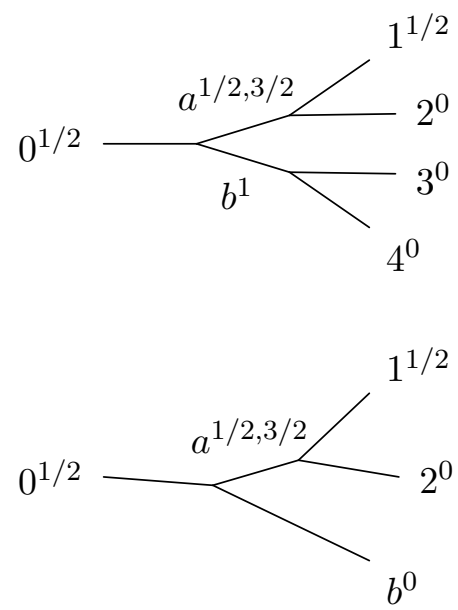

Figure 1. The eight three- and four-body decays considered in this paper. The superscripts to particles' labels specify their spins.

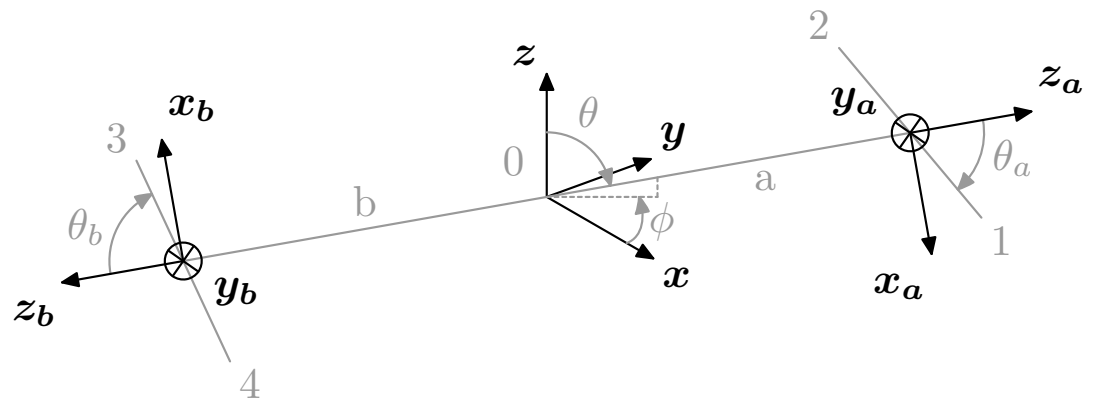

Figure 2. Reference frames defined according to the Jackson convention [4] where axes in the two daughter restframes are (anti)aligned. The azimuthal angles $\phi_{a, b}$ that are not apparent are defined in the usual way: measured from the $\boldsymbol{x}_{\boldsymbol{a}, \boldsymbol{b}}$ axes such that the $\boldsymbol{y}_{\boldsymbol{a}, \boldsymbol{b}}$ axes have $\phi_{a, b}=+\pi / 2$. Note the $a, b$ particles' momenta are pictured in the 0 particle restframe, while the 1,2 and 3,4 ones in the $a$ and $b$ restframes, respectively.

\section{Angular distributions}

The following four- and three-body decays (depicted in figure 1) will be considered:

$$
\begin{array}{rlrl}
0^{1 / 2} \longrightarrow a^{1 / 2,3 / 2} b^{1} & \longrightarrow 1^{1 / 2} 2^{0} 3^{1 / 2} 4^{1 / 2}, & 0^{1 / 2} & \longrightarrow a^{1 / 2,3 / 2} b^{1} \longrightarrow 1^{1 / 2} 2^{0} b^{1}, \\
& \longrightarrow 1^{1 / 2} 2^{0} 3^{0} 4^{0}, & \longrightarrow a^{1 / 2,3 / 2} b^{0} \longrightarrow 1^{1 / 2} 2^{0} b^{0},
\end{array}
$$

where the superscripts of particle labels specify their spins. Examples of such processes include the $\Lambda_{b} \rightarrow \Lambda J / \psi \rightarrow p \pi \mu^{+} \mu^{-}, \Lambda_{b} \rightarrow \Lambda \varphi \rightarrow p \pi K^{+} K^{-}, \Lambda_{b} \rightarrow N^{*} K_{s} \rightarrow p \pi K_{s}$ decays which were studied by the LHCb collaboration in the recent refs. [5-7], or the $\Lambda_{b} \rightarrow \Lambda(X) \gamma \rightarrow p K / \pi \gamma$ processes discussed in refs. [8-12]. Standard-model predictions relying on factorization for some charmless multibody hadronic $b$-baryon decays have been discussed in ref. [13]. $\hat{\mathrm{T}}$-odd-CP-odd asymmetries are estimated to reach approximately the twenty-percent level in $\Lambda_{b} \rightarrow N(1440) K^{-}$or $\Xi_{b} \rightarrow \Sigma^{+} K^{-}$, the percent level in the $\Lambda_{b} \rightarrow N(1440) K^{*-}$ or $\Xi_{b} \rightarrow \Sigma^{+} K^{*-}$, and the sub-percent level in $\Lambda_{b} \rightarrow \Lambda \eta^{(\prime)}$ or $\Lambda_{b} \rightarrow \Lambda \varphi$ which are dominated by one single penguin amplitude. In ref. [14], it was argued that 
new-physics contributions parametrized by effective operators (arising, e.g., from $Z^{\prime}$ or R-parity-violating supersymmetry) could be significantly larger. Additionally, the rate asymmetry in $\Lambda_{b} \rightarrow \Lambda \gamma$ was also estimated to reach at most the percent level in ref. [10].

The helicity formalism of Jakob and Wick [15] will be employed, following the so-called Jackson convention [4] for the definition of the various reference frames (see figure 2). The spins of final-state particles will be summed over. On the other hand, a nonvanishing polarisation of the initial spin- $1 / 2$ baryon will be considered. Although experimental datasets never isolate perfectly one single resonant intermediate state, the interferences between them lie beyond the scope of this work. Neither will topologies like
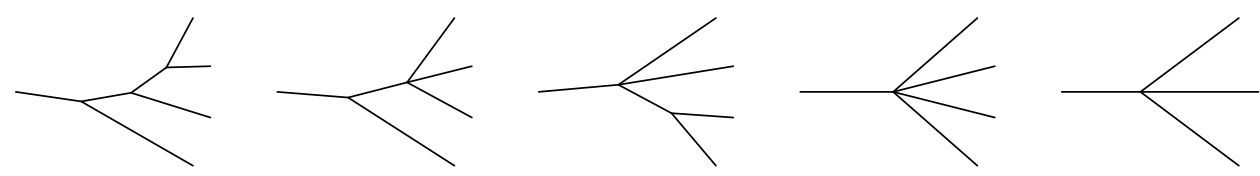

be considered.

A first $(\boldsymbol{x}, \boldsymbol{y}, \boldsymbol{z})$ system of axes is defined in the restframe of the initial - mother - particle 0 . When the production of the latter preserves parity, its polarisation vector is orthogonal to the production plane (see section $V$ of ref. [15]). To take advantage of this feature, the $\boldsymbol{z}$ axis is taken parallel to the normal of the production plane. So is a transversity frame obtained. ${ }^{1}$ Particles $a$ and $b$ are respectively produced at polar angles $\theta$ and $\pi-\theta$ from that $\boldsymbol{z}$ axis. With the spin vector of particle 0 pointing exactly in the $\boldsymbol{z}$ direction, no dependence on the azimuthal angle $\phi$ of particle $a$ is generated. The direction of the $\boldsymbol{x}$ axis is therefore chosen arbitrarily in the plane perpendicular to the $\boldsymbol{z}$ axis.

Two other systems of axes are defined in the restframes of the $a$ and $b$ daughters, as so-called helicity frames. The second one will only be relevant for four-body decays. The $\left(\boldsymbol{x}_{\boldsymbol{a}}, \boldsymbol{y}_{\boldsymbol{a}}, \boldsymbol{z}_{\boldsymbol{a}}\right)$ system is obtained by a $R(\phi, \theta, 0)^{T}$ Euler rotation $^{2}$ of the initial $(\boldsymbol{x}, \boldsymbol{y}, \boldsymbol{z})$ one, followed by a suitable boost in the $\boldsymbol{z}_{a}$ direction (parallel to particle $a$ 's momentum in the mother restframe). A $R(\phi+\pi, \pi-\theta, 0)^{T}$ rotation followed by a boost in the $\boldsymbol{z}_{\boldsymbol{b}}$ direction is required to obtain the $\left(\boldsymbol{x}_{\boldsymbol{b}}, \boldsymbol{y}_{\boldsymbol{b}}, \boldsymbol{z}_{\boldsymbol{b}}\right)$ system. Its axes are parallel or antiparallel to the $\left(\boldsymbol{x}_{\boldsymbol{a}}, \boldsymbol{y}_{\boldsymbol{a}}, \boldsymbol{z}_{\boldsymbol{a}}\right)$ ones.

The following assumptions will be made in the main text and relaxed in appendix A:

- The production of particle 0 preserves parity, so that its polarisation (if any) is aligned with the $\boldsymbol{z}$ axis. One therefore has $P_{x}=0=P_{y}$ in the density matrix for particle $0^{1 / 2}$ :

$$
\rho\left(m_{0}, m_{0}^{\prime}\right)=\frac{1}{2} \quad{ }_{-1 / 2}^{+1 / 2}\left(\begin{array}{cc}
+1 / 2 & -1 / 2 \\
1+P_{z} & P_{x}-i P_{y} \\
P_{x}+i P_{y} & 1-P_{z}
\end{array}\right)
$$

where $m_{0}^{(\prime)}$ is the component of particle 0's spin along the $\boldsymbol{z}$ axis.

\footnotetext{
${ }^{1}$ Alternatively, a helicity frame could have been obtained with $\boldsymbol{z}$ aligned to particle 0's momentum in the laboratory frame (see refs. [16-18] for some results obtained in such a frame).

${ }^{2} \mathrm{~A} R(\phi, \theta, \chi)$ transformation is the succession of three elementary rotations around the $\boldsymbol{z}, \boldsymbol{y}$, and $\boldsymbol{z}$ axes: $R_{\boldsymbol{z}}(\phi) R_{\boldsymbol{y}}(\theta) R_{\boldsymbol{z}}(\chi)$. In the so-called Jacob-Wick convention, the $\left(\boldsymbol{x}_{\boldsymbol{a}}, \boldsymbol{y}_{\boldsymbol{a}}, \boldsymbol{z}_{\boldsymbol{a}}\right)$ frame is obtained by a $R(\phi, \theta,-\phi)^{T}$ rotation of the $(\boldsymbol{x}, \boldsymbol{y}, \boldsymbol{z})$ one.
} 
- When appearing as a final-state particle, in the three-body decays we consider, the $b^{1}$ vector is taken massless so that it has no $\lambda_{b}=0$ zero helicity state (and the $A_{ \pm}$ amplitudes defined below are absent).

- The $b^{1} \rightarrow 3^{1 / 2} 4^{1 / 2}$ decay preserves parity, so that its helicity amplitudes satisfy

$$
\left|M_{b}\left(-\lambda_{3},-\lambda_{4}\right)\right|^{2}=\left|M_{b}\left(+\lambda_{3},+\lambda_{4}\right)\right|^{2} .
$$

- The $3^{1 / 2}$ and $4^{1 / 2}$ particles arising from the $b^{1}$ vector decay are massless and therefore have opposite helicities: $\lambda_{3}=-\lambda_{4}$.

In each four-body process considered here, for the $b^{1}$ decay, there is therefore one single independent combination of squared helicity amplitudes:

$$
\text { either }\left|M_{b}(1 / 2,-1 / 2)\right|^{2}+\left|M_{b}(-1 / 2,1 / 2)\right|^{2}, \quad \text { or } \quad\left|M_{b}(0,0)\right|^{2} .
$$

They will be absorbed into the definition of the $M_{0}$ helicity amplitudes for the parent $0 \rightarrow a b$ decay. We will also absorb in the $M_{0}$ 's the $\left|M_{a}(+1 / 2,0)\right|^{2}+\left|M_{a}(-1 / 2,0)\right|^{2}$ combination of $a^{1 / 2,3 / 2} \rightarrow 1^{1 / 2} 2^{0}$ amplitudes and define the

$$
\alpha_{a} \equiv \frac{\left|M_{a}(+1 / 2,0)\right|^{2}-\left|M_{a}(-1 / 2,0)\right|^{2}}{\left|M_{a}(+1 / 2,0)\right|^{2}+\left|M_{a}(-1 / 2,0)\right|^{2}}
$$

asymmetry parameter which violates parity $\mathrm{P}$. It is therefore expected to vanish if the corresponding decay proceeds through the strong interaction, like in the $\Lambda(1520) \rightarrow p K$ example of $a^{3 / 2} \rightarrow 1^{1 / 2} 2^{0}$ decay. The helicity combinations allowed for the $a, b$ system are $\left(\lambda_{a}, \lambda_{b}\right)=( \pm 1 / 2,0)$, and $( \pm 1 / 2, \pm 1)$ for a spin-1/2 particle $a^{1 / 2}$, as well as $( \pm 3 / 2, \pm 1)$ for a spin-3/2 particle $a^{3 / 2}$. We will denote the corresponding amplitudes as

$$
A_{ \pm} \equiv M_{0}( \pm 1 / 2,0), \quad B_{ \pm} \equiv M_{0}( \pm 1 / 2, \pm 1), \quad C_{ \pm} \equiv M_{0}( \pm 3 / 2, \pm 1) .
$$

As opposed to the rates of the four-body decays featuring $b^{1}$ as an intermediate particle, the three-body ones in which it appears in the final state will only contain interferences between amplitudes of identical $\lambda_{b}$. Note also that a massless $b^{1}$ vector produced onshell can only have $\lambda_{b}= \pm 1$. The $A_{ \pm}$amplitudes therefore vanish in that case. As already mentioned, this will be assumed in the main text for the three-body $0^{1 / 2} \rightarrow a^{1 / 2,3 / 2} b^{1} \rightarrow 1^{1 / 2} 2^{0} b^{1}$ decays. Finally, beyond the narrow width approximation for particles $a$ and $b$, the $A_{ \pm}, B_{ \pm}, C_{ \pm}$as well as $\alpha_{a}$ amplitudes have a non-trivial dependence on the (12) and (34) invariant masses which we will respectively denote $m_{a}$ and $m_{b}$.

The various contributions to the $\mathrm{d} \Gamma / \mathrm{d} \Omega$ angular distributions of the final-state particles for the processes depicted in figure 1 are given in table $1-8$. The overall normalisation is chosen such that the

$$
\int \mathrm{d} \Omega \equiv \int_{-1}^{+1} \frac{\mathrm{d} \cos \theta}{2} \int_{-1}^{+1} \frac{\mathrm{d} \cos \theta_{a}}{2} \int_{-1}^{+1} \frac{\mathrm{d} \cos \theta_{b}}{2} \int_{-\pi}^{+\pi} \frac{\mathrm{d} \phi}{2 \pi} \int_{-\pi}^{+\pi} \frac{\mathrm{d} \phi_{a}}{2 \pi} \int_{-\pi}^{+\pi} \frac{\mathrm{d} \phi_{b}}{2 \pi}
$$


angular integration simply yields the sum of the allowed amplitudes squared. In case all the six of them are present, one would then get

$$
\int \mathrm{d} \Omega \frac{\mathrm{d} \Gamma}{\mathrm{d} \Omega}=\left|A_{+}\right|^{2}+\left|A_{-}\right|^{2}+\left|B_{+}\right|^{2}+\left|B_{-}\right|^{2}+\left|C_{+}\right|^{2}+\left|C_{-}\right|^{2} .
$$

Here, again, the dependence on the $m_{a, b}$ invariant masses is kept implicit.

Some of the angular distributions we obtained have already been presented elsewhere, sometimes partially only. table 1 agrees with the table 1 of ref. [19]. table 5 agrees with eq. (7) of ref. [11]. The terms in table 6 that are not proportional to $\alpha_{a}$ only match eq. (15) of ref. [11], provided $w_{5}$ and $w_{6}$ defined there are respectively multiplied by factors of $\pm P_{z}$. The relative sign between these $B_{+} C_{+}$and $B_{-} C_{-}$interferences can be understood given the $d_{-\mu,-\lambda}^{j}(\theta)=(-1)^{\lambda-\mu} d_{\mu, \lambda}^{j}(\theta)$ symmetry relations between Wigner matrices (see eq. (A1) of ref. [15]). Note also a relative complex conjugation of the amplitudes defined here and there, as well as the use of the Jacob-Wick convention there which leads to expressions identical to the ones we obtain with the Jackson convention for the terms compared when $\phi_{\Lambda}$ is set to 0 there. Both table 7 and the $A_{ \pm}$dependence of table 3 agree with eq. (16) and (21) of ref. [20], obtained with $P_{z}=+1$.

\section{Discrete symmetry properties}

To establish the parity $\mathrm{P}$ and motion reversal $\hat{\mathrm{T}}$ transformation properties of the various contributions to the differential distributions displayed in table 1-8, let us define our kinematic variables and axes in terms of physical momenta.

In the restframe of particle 0 , let us assume that the production plane is defined by the momenta $\boldsymbol{p}_{\boldsymbol{A}}$ and $\boldsymbol{p}_{\boldsymbol{B}}$ of two of the particles involved. One can then construct the $(\boldsymbol{x}, \boldsymbol{y}, \boldsymbol{z})$ frame as

$$
x=\frac{p_{A}}{\left|p_{A}\right|}, \quad z=\frac{p_{A} \times p_{B}}{\left|p_{A} \times p_{B}\right|}, \quad y=z \times x .
$$

The $\boldsymbol{z}$ axis is thus a P-even- $\hat{\mathrm{T}}$-even pseudovector, while $\boldsymbol{x}$ and $\boldsymbol{y}$ are both P-odd- $\hat{\mathrm{T}}$-odd vectors. The $\left(\boldsymbol{x}_{\boldsymbol{a}}, \boldsymbol{y}_{\boldsymbol{a}}, \boldsymbol{z}_{\boldsymbol{a}}\right)$ system is then obtained as

$$
z_{a}=\frac{p_{1}+p_{2}}{\left|p_{1}+p_{2}\right|}, \quad y_{a}=\frac{z \times z_{a}}{\left|z \times z_{a}\right|}, \quad x_{a}=\frac{y_{a} \times z_{a}}{\left|y_{a} \times z_{a}\right|},
$$

where $\boldsymbol{p}_{\mathbf{1}}$ and $\boldsymbol{p}_{\mathbf{2}}$ are the momenta of particles 1 and 2 in particle 0's restframe. It follows

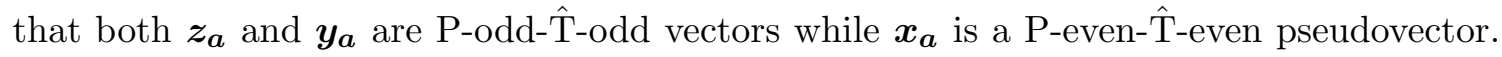
Similar conclusions hold for $\left(\boldsymbol{x}_{\boldsymbol{b}}, \boldsymbol{y}_{\boldsymbol{b}}, \boldsymbol{z}_{\boldsymbol{b}}\right)=\left(-\boldsymbol{x}_{\boldsymbol{a}}, \boldsymbol{y}_{\boldsymbol{a}},-\boldsymbol{z}_{\boldsymbol{a}}\right)$. The polar $\theta$ and azimuthal $\phi$ angles can be obtained from the equalities:

$\cos \theta=\boldsymbol{z} \cdot \boldsymbol{z}_{\boldsymbol{a}}, \quad \sin \theta=+\sqrt{1-\cos ^{2} \theta}, \quad \cos \phi=\left(\boldsymbol{z} \times \boldsymbol{z}_{\boldsymbol{a}}\right) \cdot \boldsymbol{y}, \quad \sin \phi=-\left(\boldsymbol{z} \times \boldsymbol{z}_{\boldsymbol{a}}\right) \cdot \boldsymbol{x}$, which establish that $\cos \theta$ is a $\mathrm{P}$-odd- $\hat{\mathrm{T}}$-odd kinematic variable, while $\sin \theta, \cos \phi$, and $\sin \phi$ are $\mathrm{P}$-even- $\hat{\mathrm{T}}$-even. Moreover, defining the $\mathrm{P}$-even- $\hat{\mathrm{T}}$-even pseudovectors

$$
n_{a}=\frac{p_{1} \times p_{2}}{\left|p_{1} \times p_{2}\right|}, \quad n_{b}=\frac{p_{3} \times p_{4}}{\left|p_{3} \times p_{4}\right|},
$$




\begin{tabular}{|c|c|c|c|c|c|c|}
\hline$+3 / 2$ & $\left|A_{+}\right|^{2}+\left|A_{-}\right|^{2}$ & & & & $\sin ^{2} \theta_{b}$ & \\
\hline$+3 / 4$ & $\left|B_{+}\right|^{2}+\left|B_{-}\right|^{2}$ & & & & $1+\cos ^{2} \theta_{b}$ & \\
\hline$+3 / 2$ & $\left|A_{+}\right|^{2}-\left|A_{-}\right|^{2}$ & $\alpha_{a}$ & & $\cos \theta_{a}$ & $\sin ^{2} \theta_{b}$ & \\
\hline$+3 / 4$ & $\left|B_{+}\right|^{2}-\left|B_{-}\right|^{2}$ & $\alpha_{a}$ & & $\cos \theta_{a}$ & $1+\cos ^{2} \theta_{b}$ & \\
\hline$-3 / 2 \sqrt{2}$ & $\operatorname{Re}\left\{A_{+}^{*} B_{-}\right\}-\operatorname{Re}\left\{A_{-}^{*} B_{+}\right\}$ & $\alpha_{a}$ & & $\sin \theta_{a}$ & $\sin 2 \theta_{b}$ & $\cos \left(\phi_{a}+\phi_{b}\right)$ \\
\hline$+3 / 2$ & $\left|A_{+}\right|^{2}-\left|A_{-}\right|^{2}$ & & $P_{z} \quad \cos \theta$ & & $\sin ^{2} \theta_{b}$ & \\
\hline$-3 / 4$ & $\left|B_{+}\right|^{2}-\left|B_{-}\right|^{2}$ & & $P_{z} \quad \cos \theta$ & & $1+\cos ^{2} \theta_{b}$ & \\
\hline$-3 / 2 \sqrt{2}$ & $\operatorname{Re}\left\{A_{+}^{*} B_{+}\right\}-\operatorname{Re}\left\{A_{-}^{*} B_{-}\right\}$ & & $P_{z} \quad \sin \theta$ & & $\sin 2 \theta_{b}$ & $\cos \phi_{b}$ \\
\hline$+3 / 2$ & $\left|A_{+}\right|^{2}+\left|A_{-}\right|^{2}$ & $\alpha_{a}$ & $P_{z} \quad \cos \theta$ & $\cos \theta_{a}$ & $\sin ^{2} \theta_{b}$ & \\
\hline$-3 / 4$ & $\left|B_{+}\right|^{2}+\left|B_{-}\right|^{2}$ & $\alpha_{a}$ & $P_{z} \quad \cos \theta$ & $\cos \theta_{a}$ & $1+\cos ^{2} \theta_{b}$ & \\
\hline$-3 / 2 \sqrt{2}$ & $\operatorname{Re}\left\{A_{+}^{*} B_{-}\right\}+\operatorname{Re}\left\{A_{-}^{*} B_{+}\right\}$ & $\alpha_{a}$ & $P_{z} \quad \cos \theta$ & $\sin \theta_{a}$ & $\sin 2 \theta_{b}$ & $\cos \left(\phi_{a}+\phi_{b}\right)$ \\
\hline$-3 / 2 \sqrt{2}$ & $\operatorname{Re}\left\{A_{+}^{*} B_{+}\right\}+\operatorname{Re}\left\{A_{-}^{*} B_{-}\right\}$ & $\alpha_{a}$ & $P_{z} \quad \sin \theta$ & $\cos \theta_{a}$ & $\sin 2 \theta_{b}$ & $\cos \phi_{b}$ \\
\hline-3 & $\operatorname{Re}\left\{A_{+}^{*} A_{-}\right\}$ & $\alpha_{a}$ & $P_{z} \quad \sin \theta$ & $\sin \theta_{a}$ & $\sin ^{2} \theta_{b}$ & $\cos \phi_{a}$ \\
\hline$-3 / 2$ & $\operatorname{Re}\left\{B_{+}^{*} B_{-}\right\}$ & $\alpha_{a}$ & $P_{z} \quad \sin \theta$ & $\sin \theta_{a}$ & $\sin ^{2} \theta_{b}$ & $\cos \left(\phi_{a}+2 \phi_{b}\right)$ \\
\hline$+3 / 2 \sqrt{2}$ & $\operatorname{Im}\left\{A_{+}^{*} B_{+}\right\}+\operatorname{Im}\left\{A_{-}^{*} B_{-}\right\}$ & & $P_{z} \quad \sin \theta$ & & $\sin 2 \theta_{b}$ & $\sin \phi_{b}$ \\
\hline$-3 / 2 \sqrt{2}$ & $\operatorname{Im}\left\{A_{+}^{*} B_{-}\right\}-\operatorname{Im}\left\{A_{-}^{*} B_{+}\right\}$ & $\alpha_{a}$ & $P_{z} \quad \cos \theta$ & $\sin \theta_{a}$ & $\sin 2 \theta_{b}$ & $\sin \left(\phi_{a}+\phi_{b}\right)$ \\
\hline$+3 / 2 \sqrt{2}$ & $\operatorname{Im}\left\{A_{+}^{*} B_{+}\right\}-\operatorname{Im}\left\{A_{-}^{*} B_{-}\right\}$ & $\alpha_{a}$ & $P_{z} \quad \sin \theta$ & $\cos \theta_{a}$ & $\sin 2 \theta_{b}$ & $\sin \phi_{b}$ \\
\hline-3 & $\operatorname{Im}\left\{A_{+}^{*} A_{-}\right\}$ & $\alpha_{a}$ & $P_{z} \quad \sin \theta$ & $\sin \theta_{a}$ & $\sin ^{2} \theta_{b}$ & $\sin \phi_{a}$ \\
\hline$-3 / 2$ & $\operatorname{Im}\left\{B_{+}^{*} B_{-}\right\}$ & $\alpha_{a}$ & $P_{z} \quad \sin \theta$ & $\sin \theta_{a}$ & $\sin ^{2} \theta_{b}$ & $\sin \left(\phi_{a}+2 \phi_{b}\right)$ \\
\hline$-3 / 2 \sqrt{2}$ & $\operatorname{Im}\left\{A_{+}^{*} B_{-}\right\}+\operatorname{Im}\left\{A_{-}^{*} B_{+}\right\}$ & $\alpha_{a}$ & & $\sin \theta_{a}$ & $\sin 2 \theta_{b}$ & $\sin \left(\phi_{a}+\phi_{b}\right)$ \\
\hline
\end{tabular}

Table 1. Various contributions to the angular distribution of the $0^{1 / 2} \rightarrow a^{1 / 2} b^{1} \rightarrow 1^{1 / 2} 2^{0} 3^{1 / 2} 4^{1 / 2}$ process, with conventions and assumptions specified in the text. Each line corresponds to a term of different angular dependence (most of them being independent). The separation in columns is only meant to ease the comparison between the various factors appearing in each term. The four blocks distinguish terms whose combinations of angular and polarisation dependence have different parity and motion reversal transformation properties. They are respectively P-even- $\hat{\mathrm{T}}$-even, $\mathrm{P}$-odd- $\hat{\mathrm{T}}$-even, P-even- $\hat{T}$-odd, and P-odd- $\hat{T}$-odd. 


\begin{tabular}{|c|c|c|c|c|c|c|}
\hline$+3 / 8$ & $\left|B_{+}\right|^{2}+\left|B_{-}\right|^{2}+3\left|C_{+}\right|^{2}+3\left|C_{-}\right|^{2}$ & & & & $1+\cos ^{2} \theta_{b}$ & \\
\hline $\begin{array}{l}-3 \sqrt{2 / 3} / 4 \\
\quad+3 / 4\end{array}$ & $\begin{array}{c}\operatorname{Re}\left\{A_{+}^{*} C_{+}\right\}+\operatorname{Re}\left\{A_{-}^{*} C_{-}\right\} \\
\left|A_{+}\right|^{2}+\left|A_{-}\right|^{2}\end{array}$ & & & $\begin{array}{c}\sin 2 \theta_{a} \\
\left(1+3 \cos ^{2} \theta_{a}\right)\end{array}$ & $\begin{array}{l}\sin 2 \theta_{b} \\
\sin ^{2} \theta_{b}\end{array}$ & $\cos \left(\phi_{a}+\phi_{b}\right)$ \\
\hline$-3 \sqrt{3} / 4$ & $\operatorname{Re}\left\{B_{+}^{*} C_{-}\right\}+\operatorname{Re}\left\{B_{-}^{*} C_{+}\right\}$ & & & $\sin ^{2} \theta_{a}$ & $\sin ^{2} \theta_{b}$ & $\cos \left(2 \phi_{a}+2 \phi_{b}\right)$ \\
\hline$+9 / 8$ & $\left|B_{+}\right|^{2}+\left|B_{-}\right|^{2}-\left|C_{+}\right|^{2}-\left|C_{-}\right|^{2}$ & & & $\cos ^{2} \theta_{a}$ & $1+\cos ^{2} \theta_{b}$ & \\
\hline$-3 / 8$ & $5\left|B_{+}\right|^{2}-5\left|B_{-}\right|^{2}-3\left|C_{+}\right|^{2}+3\left|C_{-}\right|^{2}$ & $\alpha_{a}$ & & $\cos \theta_{a}$ & $1+\cos ^{2} \theta_{b}$ & \\
\hline$-3 / 4$ & $\left|A_{+}\right|^{2}-\left|A_{-}\right|^{2}$ & $\alpha_{a}$ & & $\left(5-9 \cos ^{2} \theta_{a}\right) \cos \theta_{a}$ & $\sin ^{2} \theta_{b}$ & \\
\hline$+3 \sqrt{2 / 3} / 4$ & $\operatorname{Re}\left\{A_{+}^{*} C_{+}\right\}-\operatorname{Re}\left\{A_{-}^{*} C_{-}\right\}$ & $\alpha_{a}$ & & $\left(1-3 \cos ^{2} \theta_{a}\right) \sin \theta_{a}$ & $\sin 2 \theta_{b}$ & $\cos \left(\phi_{a}+\phi_{b}\right)$ \\
\hline$+3 / 4 \sqrt{2}$ & $\operatorname{Re}\left\{A_{+}^{*} B_{-}\right\}-\operatorname{Re}\left\{A_{-}^{*} B_{+}\right\}$ & $\alpha_{a}$ & & $\left(1-9 \cos ^{2} \theta_{a}\right) \sin \theta_{a}$ & $\sin 2 \theta_{b}$ & $\cos \left(\phi_{a}+\phi_{b}\right)$ \\
\hline$+9 \sqrt{3} / 4$ & $\operatorname{Re}\left\{B_{+}^{*} C_{-}\right\}-\operatorname{Re}\left\{B_{-}^{*} C_{+}\right\}$ & $\alpha_{a}$ & & $\sin ^{2} \theta_{a} \cos \theta_{a}$ & $\sin ^{2} \theta_{b}$ & $\cos \left(2 \phi_{a}+2 \phi_{b}\right)$ \\
\hline$+9 / 8$ & $3\left|B_{+}\right|^{2}-3\left|B_{-}\right|^{2}-\left|C_{+}\right|^{2}+\left|C_{-}\right|^{2}$ & $\alpha_{a}$ & & $\cos ^{3} \theta_{a}$ & $1+\cos ^{2} \theta_{b}$ & \\
\hline$-3 / 8$ & $\left|B_{+}\right|^{2}-\left|B_{-}\right|^{2}-3\left|C_{+}\right|^{2}+3\left|C_{-}\right|^{2}$ & & $P_{z} \cos \theta$ & & $1+\cos ^{2} \theta_{b}$ & \\
\hline$-3 \sqrt{2 / 3} / 4$ & $\operatorname{Re}\left\{A_{+}^{*} C_{+}\right\}-\operatorname{Re}\left\{A_{-}^{*} C_{-}\right\}$ & & $P_{z} \cos \theta$ & $\sin 2 \theta_{a}$ & $\sin 2 \theta_{b}$ & $\cos \left(\phi_{a}+\phi_{b}\right)$ \\
\hline$+3 / 4$ & $\left|A_{+}\right|^{2}-\left|A_{-}\right|^{2}$ & & $P_{z} \cos \theta$ & $\left(1+3 \cos ^{2} \theta_{a}\right)$ & $\sin ^{2} \theta_{b}$ & \\
\hline$+3 \sqrt{3} / 4$ & $\operatorname{Re}\left\{B_{+}^{*} C_{-}\right\}-\operatorname{Re}\left\{B_{-}^{*} C_{+}\right\}$ & & $P_{z} \cos \theta$ & $\sin ^{2} \theta_{a}$ & $\sin ^{2} \theta_{b}$ & $\cos \left(2 \phi_{a}+2 \phi_{b}\right)$ \\
\hline$-9 / 8$ & $\left|B_{+}\right|^{2}-\left|B_{-}\right|^{2}+\left|C_{+}\right|^{2}-\left|C_{-}\right|^{2}$ & & $P_{z} \cos \theta$ & $\cos ^{2} \theta_{a}$ & $1+\cos ^{2} \theta_{b}$ & \\
\hline$+3 \sqrt{3} / 4$ & $\operatorname{Re}\left\{B_{+}^{*} C_{+}\right\}-\operatorname{Re}\left\{B_{-}^{*} C_{-}\right\}$ & & $P_{z} \sin \theta$ & $\sin 2 \theta_{a}$ & $1+\cos ^{2} \theta_{b}$ & $\cos \phi_{a}$ \\
\hline$-3 / 4 \sqrt{2}$ & $\operatorname{Re}\left\{A_{+}^{*} B_{+}\right\}-\operatorname{Re}\left\{A_{-}^{*} B_{-}\right\}$ & & $P_{z} \sin \theta$ & $\left(1+3 \cos ^{2} \theta_{a}\right)$ & $\sin 2 \theta_{b}$ & $\phi_{b}$ \\
\hline$-3 \sqrt{2 / 3} / 4$ & $\operatorname{Re}\left\{A_{+}^{*} C_{-}\right\}-\operatorname{Re}\left\{A_{-}^{*} C_{+}\right\}$ & & $P_{z} \sin \theta$ & $\sin ^{2} \theta_{a}$ & $\sin 2 \theta_{b}$ & $\cos \left(2 \phi_{a}+\phi_{b}\right)$ \\
\hline$+3 / 8$ & $5\left|B_{+}\right|^{2}+5\left|B_{-}\right|^{2}+3\left|C_{+}\right|^{2}+3\left|C_{-}\right|^{2}$ & $\alpha_{a}$ & $P_{z} \cos \theta$ & $\cos \theta_{a}$ & $1+\cos ^{2} \theta_{b}$ & \\
\hline$-3 / 4$ & $\left|A_{+}\right|^{2}+\left|A_{-}\right|^{2}$ & $\alpha_{a}$ & $P_{z} \cos \theta$ & $\left(5-9 \cos ^{2} \theta_{a}\right) \cos \theta_{a}$ & $\sin ^{2} \theta_{b}$ & \\
\hline$+3 \sqrt{2 / 3} / 4$ & $\operatorname{Re}\left\{A_{+}^{*} C_{+}\right\}+\operatorname{Re}\left\{A_{-}^{*} C_{-}\right\}$ & $\alpha_{a}$ & $P_{z} \cos \theta$ & $\left(1-3 \cos ^{2} \theta_{a}\right) \sin \theta_{a}$ & $\sin 2 \theta_{b}$ & $\cos \left(\phi_{a}+\phi_{b}\right)$ \\
\hline$+3 / 4 \sqrt{2}$ & $\operatorname{Re}\left\{A_{+}^{*} B_{-}\right\}+\operatorname{Re}\left\{A_{-}^{*} B_{+}\right\}$ & $\alpha_{a}$ & $P_{z} \cos \theta$ & $\left(1-9 \cos ^{2} \theta_{a}\right) \sin \theta_{a}$ & $\sin 2 \theta_{b}$ & $\cos \left(\phi_{a}+\phi_{b}\right)$ \\
\hline$-9 \sqrt{3} / 4$ & $\operatorname{Re}\left\{B_{+}^{*} C_{-}\right\}+\operatorname{Re}\left\{B_{-}^{*} C_{+}\right\}$ & $\alpha_{a}$ & $P_{z} \cos \theta$ & $\sin ^{2} \theta_{a} \cos \theta_{a}$ & $\sin ^{2} \theta_{b}$ & $\cos \left(2 \phi_{a}+2 \phi_{b}\right)$ \\
\hline$-9 / 8$ & $3\left|B_{+}\right|^{2}+3\left|B_{-}\right|^{2}+\left|C_{+}\right|^{2}+\left|C_{-}\right|^{2}$ & $\alpha_{a}$ & $P_{z} \cos \theta$ & $\cos ^{3} \theta_{a}$ & $1+\cos ^{2} \theta_{b}$ & \\
\hline$+3 / 4 \sqrt{2}$ & $\operatorname{Re}\left\{A_{+}^{*} B_{+}\right\}+\operatorname{Re}\left\{A_{-}^{*} B_{-}\right\}$ & $\alpha_{a}$ & $P_{z} \sin \theta$ & $\left(5-9 \cos ^{2} \theta_{a}\right) \cos \theta_{a}$ & $\sin 2 \theta_{b}$ & $\cos \phi_{b}$ \\
\hline$-3 \sqrt{3} / 4$ & $\operatorname{Re}\left\{B_{+}^{*} C_{+}\right\}+\operatorname{Re}\left\{B_{-}^{*} C_{-}\right\}$ & $\alpha_{a}$ & $P_{z} \sin \theta$ & $\left(1-3 \cos ^{2} \theta_{a}\right) \sin \theta_{a}$ & $1+\cos ^{2} \theta_{b}$ & $\cos \phi_{a}$ \\
\hline$+3 / 2$ & $\operatorname{Re}\left\{A_{+}^{*} A_{-}\right\}$ & $\alpha_{a}$ & $P_{z} \sin \theta$ & $\left(1-9 \cos ^{2} \theta_{a}\right) \sin \theta_{a}$ & $\sin ^{2} \theta_{b}$ & $\cos \phi_{a}$ \\
\hline$+3 / 4$ & $\operatorname{Re}\left\{B_{+}^{*} B_{-}\right\}$ & $\alpha_{a}$ & $P_{z} \sin \theta$ & $\left(1-9 \cos ^{2} \theta_{a}\right) \sin \theta_{a}$ & $\sin ^{2} \theta_{b}$ & $\cos \left(\phi_{a}+2 \phi_{b}\right)$ \\
\hline$+9 / 4$ & $\operatorname{Re}\left\{C_{+}^{*} C_{-}\right\}$ & $\alpha_{a}$ & $P_{z} \sin \theta$ & $\sin ^{3} \theta_{a}$ & $\sin ^{2} \theta_{b}$ & $\cos \left(3 \phi_{a}+2 \phi_{b}\right)$ \\
\hline$+9 \sqrt{2 / 3} / 4$ & $\operatorname{Re}\left\{A_{+}^{*} C_{-}\right\}+\operatorname{Re}\left\{A_{-}^{*} C_{+}\right\}$ & $\alpha_{a}$ & $P_{z} \sin \theta$ & $\sin ^{2} \theta_{a} \cos \theta_{a}$ & $\sin 2 \theta_{b}$ & $\cos \left(2 \phi_{a}+\phi_{b}\right)$ \\
\hline$+3 \sqrt{2 / 3} / 4$ & $\operatorname{Im}\left\{A_{+}^{*} C_{+}\right\}+\operatorname{Im}\left\{A_{-}^{*} C_{-}\right\}$ & & $P_{z} \cos \theta$ & $\sin 2 \theta_{a}$ & $\sin 2 \theta_{b}$ & $\sin \left(\phi_{a}+\phi_{b}\right)$ \\
\hline$+3 \sqrt{3} / 4$ & $\operatorname{Im}\left\{B_{+}^{*} C_{-}\right\}+\operatorname{Im}\left\{B_{-}^{*} C_{+}\right\}$ & & $P_{z} \cos \theta$ & $\sin ^{2} \theta_{a}$ & $\sin ^{2} \theta_{b}$ & $\sin \left(2 \phi_{a}+2 \phi_{b}\right)$ \\
\hline$-3 \sqrt{3} / 4$ & $\operatorname{Im}\left\{B_{+}^{*} C_{+}\right\}+\operatorname{Im}\left\{B_{-}^{*} C_{-}\right\}$ & & $P_{z} \sin \theta$ & $\sin 2 \theta_{a}$ & $1+\cos ^{2} \theta_{b}$ & $\sin \phi_{a}$ \\
\hline$+3 / 4 \sqrt{2}$ & $\operatorname{Im}\left\{A_{+}^{*} B_{+}\right\}+\operatorname{Im}\left\{A_{-}^{*} B_{-}\right\}$ & & $P_{z} \sin \theta$ & $\left(1+3 \cos ^{2} \theta_{a}\right)$ & $\sin 2 \theta_{b}$ & $\sin \phi_{b}$ \\
\hline$-3 \sqrt{2 / 3} / 4$ & $\operatorname{Im}\left\{A_{+}^{*} C_{-}\right\}+\operatorname{Im}\left\{A_{-}^{*} C_{+}\right\}$ & & $P_{z} \sin \theta$ & $\sin ^{2} \theta_{a}$ & $\sin 2 \theta_{b}$ & $\sin \left(2 \phi_{a}+\phi_{b}\right)$ \\
\hline$-3 \sqrt{2 / 3} / 4$ & $\operatorname{Im}\left\{A_{+}^{*} C_{+}\right\}-\operatorname{Im}\left\{A_{-}^{*} C_{-}\right\}$ & & $P_{z} \cos \theta$ & $\left(1-3 \cos ^{2} \theta_{a}\right) \sin \theta_{a}$ & $\sin 2 \theta_{b}$ & $\sin \left(\phi_{a}+\phi_{b}\right)$ \\
\hline$+3 / 4 \sqrt{2}$ & $\operatorname{Im}\left\{A_{+}^{*} B_{-}\right\}-\operatorname{Im}\left\{A_{-}^{*} B_{+}\right\}$ & & $P_{z} \cos \theta$ & $\left(1-9 \cos ^{2} \theta_{a}\right) \sin \theta_{a}$ & $\sin 2 \theta_{b}$ & $\sin \left(\phi_{a}+\phi_{b}\right)$ \\
\hline$-9 \sqrt{3} / 4$ & $\operatorname{Im}\left\{B_{+}^{*} C_{-}\right\}-\operatorname{Im}\left\{B_{-}^{*} C_{+}\right\}$ & $\alpha_{a}$ & $P_{z} \cos \theta$ & $\sin ^{2} \theta_{a} \cos \theta_{a}$ & $\sin ^{2} \theta_{b}$ & $\sin \left(2 \phi_{a}+2 \phi_{b}\right)$ \\
\hline$-3 / 4 \sqrt{2}$ & $\operatorname{Im}\left\{A_{+}^{*} B_{+}\right\}-\operatorname{Im}\left\{A_{-}^{*} B_{-}\right\}$ & $\alpha_{a}$ & $P_{z} \sin \theta$ & $\left(5-9 \cos ^{2} \theta_{a}\right) \cos \theta_{a}$ & $\sin 2 \theta_{b}$ & $\sin \phi_{b}$ \\
\hline$+3 \sqrt{3} / 4$ & $\operatorname{Im}\left\{B_{+}^{*} C_{+}\right\}-\operatorname{Im}\left\{B_{-}^{*} C_{-}\right\}$ & $\alpha_{a}$ & $P_{z} \sin \theta$ & $\left(1-3 \cos ^{2} \theta_{a}\right) \sin \theta_{a}$ & $1+\cos ^{2} \theta_{b}$ & $\sin \phi_{a}$ \\
\hline$+3 / 2$ & $\operatorname{Im}\left\{A_{+}^{*} A_{-}\right\}$ & $\alpha_{a}$ & $P_{z} \sin \theta$ & $\left(1-9 \cos ^{2} \theta_{a}\right) \sin \theta_{a}$ & $\sin ^{2} \theta_{b}$ & $\sin \phi_{a}$ \\
\hline$+3 / 4$ & $\operatorname{Im}\left\{B_{+}^{*} B_{-}\right\}$ & $\alpha_{a}$ & $P_{z} \sin \theta$ & $\left(1-9 \cos ^{2} \theta_{a}\right) \sin \theta_{a}$ & $\sin ^{2} \theta_{b}$ & $\sin \left(\phi_{a}+2 \phi_{b}\right)$ \\
\hline$+9 / 4$ & $\operatorname{Im}\left\{C_{+}^{*} C_{-}\right\}$ & $\alpha_{a}$ & $P_{z} \sin \theta$ & $\sin ^{3} \theta_{a}$ & $\sin ^{2} \theta_{b}$ & $\sin \left(3 \phi_{a}+2 \phi_{b}\right)$ \\
\hline$+9 \sqrt{2 / 3} / 4$ & $\operatorname{Im}\left\{A_{+}^{*} C_{-}\right\}-\operatorname{Im}\left\{A_{-}^{*} C_{+}\right\}$ & $\alpha_{a}$ & $P_{z} \sin \theta$ & $\sin ^{2} \theta_{a} \cos \theta_{a}$ & $\sin 2 \theta_{b}$ & $\sin \left(2 \phi_{a}+\phi_{b}\right)$ \\
\hline$+3 \sqrt{2 / 3} / 4$ & $\operatorname{Im}\left\{A_{+}^{*} C_{+}\right\}-\operatorname{Im}\left\{A_{-}^{*} C_{-}\right\}$ & & & $\sin 2 \theta_{a}$ & $\sin 2 \theta_{b}$ & $\sin \left(\phi_{a}+\phi_{b}\right)$ \\
\hline$-3 \sqrt{3} / 4$ & $\operatorname{Im}\left\{B_{+}^{*} C_{-}\right\}-\operatorname{Im}\left\{B_{-}^{*} C_{+}\right\}$ & & & $\sin ^{2} \theta_{a}$ & $\sin ^{2} \theta_{b}$ & $\sin \left(2 \phi_{a}+2 \phi_{b}\right)$ \\
\hline$-3 \sqrt{2 / 3} / 4$ & $\operatorname{Im}\left\{A_{+}^{*} C_{+}\right\}+\operatorname{Im}\left\{A_{-}^{*} C_{-}\right\}$ & $\alpha_{a}$ & & $\left(1-3 \cos ^{2} \theta_{a}\right) \sin \theta_{a}$ & $\sin 2 \theta_{b}$ & $\sin \left(\phi_{a}+\phi_{b}\right)$ \\
\hline$+3 / 4 \sqrt{2}$ & $\operatorname{Im}\left\{A_{+}^{*} B_{-}\right\}+\operatorname{Im}\left\{A_{-}^{*} B_{+}\right\}$ & $\alpha_{a}$ & & $\left(1-9 \cos ^{2} \theta_{a}\right) \sin \theta_{a}$ & $\sin 2 \theta_{b}$ & $\sin \left(\phi_{a}+\phi_{b}\right)$ \\
\hline$+9 \sqrt{3} / 4$ & $\operatorname{Im}\left\{B_{+}^{*} C_{-}\right\}+\operatorname{Im}\left\{B_{-}^{*} C_{+}\right\}$ & $\alpha_{a}$ & & $\sin ^{2} \theta_{a} \cos \theta_{a}$ & $\sin ^{2} \theta_{b}$ & $\sin \left(2 \phi_{a}+2 \phi_{b}\right)$ \\
\hline
\end{tabular}

Table 2. Same as table 1 , for the $0^{1 / 2} \rightarrow a^{3 / 2} b^{1} \rightarrow 1^{1 / 2} 2^{0} 3^{1 / 2} 4^{1 / 2}$ process where particle $a$ has spin $3 / 2$ instead of $1 / 2$. 


\begin{tabular}{|c|c|c|c|c|c|c|}
\hline+3 & $\left|A_{+}\right|^{2}+\left|A_{-}\right|^{2}$ & & & & $\cos ^{2} \theta_{b}$ & \\
\hline$+3 / 2$ & $\left|B_{+}\right|^{2}+\left|B_{-}\right|^{2}$ & & & & $\sin ^{2} \theta_{b}$ & \\
\hline+3 & $\left|A_{+}\right|^{2}-\left|A_{-}\right|^{2}$ & $\alpha_{a}$ & & $\cos \theta_{a}$ & $\cos ^{2} \theta_{b}$ & \\
\hline$+3 / 2$ & $\left|B_{+}\right|^{2}-\left|B_{-}\right|^{2}$ & $\alpha_{a}$ & & $\cos \theta_{a}$ & $\sin ^{2} \theta_{b}$ & \\
\hline$+3 / \sqrt{2}$ & $\operatorname{Re}\left\{A_{+}^{*} B_{-}\right\}-\operatorname{Re}\left\{A_{-}^{*} B_{+}\right\}$ & $\alpha_{a}$ & & $\sin \theta_{a}$ & $\sin 2 \theta_{b}$ & $\cos \left(\phi_{a}+\phi_{b}\right)$ \\
\hline+3 & $\left|A_{+}\right|^{2}-\left|A_{-}\right|^{2}$ & & $P_{z} \quad \cos \theta$ & & $\cos ^{2} \theta_{b}$ & \\
\hline$-3 / 2$ & $\left|B_{+}\right|^{2}-\left|B_{-}\right|^{2}$ & & $P_{z} \quad \cos \theta$ & & $\sin ^{2} \theta_{b}$ & \\
\hline$+3 / \sqrt{2}$ & $\operatorname{Re}\left\{A_{+}^{*} B_{+}\right\}-\operatorname{Re}\left\{A_{-}^{*} B_{-}\right\}$ & & $P_{z} \quad \sin \theta$ & & $\sin 2 \theta_{b}$ & $\cos \phi_{b}$ \\
\hline+3 & $\left|A_{+}\right|^{2}+\left|A_{-}\right|^{2}$ & $\alpha_{a}$ & $P_{z} \quad \cos \theta$ & $\cos \theta_{a}$ & $\cos ^{2} \theta_{b}$ & \\
\hline$-3 / 2$ & $\left|B_{+}\right|^{2}+\left|B_{-}\right|^{2}$ & $\alpha_{a}$ & $P_{z} \quad \cos \theta$ & $\cos \theta_{a}$ & $\sin ^{2} \theta_{b}$ & \\
\hline$+3 / \sqrt{2}$ & $\operatorname{Re}\left\{A_{+}^{*} B_{-}\right\}+\operatorname{Re}\left\{A_{-}^{*} B_{+}\right\}$ & $\alpha_{a}$ & $P_{z} \quad \cos \theta$ & $\sin \theta_{a}$ & $\sin 2 \theta_{b}$ & $\cos \left(\phi_{a}+\phi_{b}\right)$ \\
\hline$+3 / \sqrt{2}$ & $\operatorname{Re}\left\{A_{+}^{*} B_{+}\right\}+\operatorname{Re}\left\{A_{-}^{*} B_{-}\right\}$ & $\alpha_{a}$ & $P_{z} \quad \sin \theta$ & $\cos \theta_{a}$ & $\sin 2 \theta_{b}$ & $\cos \phi_{b}$ \\
\hline-6 & $\operatorname{Re}\left\{A_{+}^{*} A_{-}\right\}$ & $\alpha_{a}$ & $P_{z} \quad \sin \theta$ & $\sin \theta_{a}$ & $\cos ^{2} \theta_{b}$ & $\cos \phi_{a}$ \\
\hline+3 & $\operatorname{Re}\left\{B_{+}^{*} B_{-}\right\}$ & $\alpha_{a}$ & $P_{z} \quad \sin \theta$ & $\sin \theta_{a}$ & $\sin ^{2} \theta_{b}$ & $\cos \left(\phi_{a}+2 \phi_{b}\right)$ \\
\hline$-3 / \sqrt{2}$ & $\operatorname{Im}\left\{A_{+}^{*} B_{+}\right\}+\operatorname{Im}\left\{A_{-}^{*} B_{-}\right\}$ & & $P_{z} \quad \sin \theta$ & & $\sin 2 \theta_{b}$ & $\sin \phi_{b}$ \\
\hline$+3 / \sqrt{2}$ & $\operatorname{Im}\left\{A_{+}^{*} B_{-}\right\}-\operatorname{Im}\left\{A_{-}^{*} B_{+}\right\}$ & $\alpha_{a}$ & $P_{z} \quad \cos \theta$ & $\sin \theta_{a}$ & $\sin 2 \theta_{b}$ & $\sin \left(\phi_{a}+\phi_{b}\right)$ \\
\hline$-3 / \sqrt{2}$ & $\operatorname{Im}\left\{A_{+}^{*} B_{+}\right\}-\operatorname{Im}\left\{A_{-}^{*} B_{-}\right\}$ & $\alpha_{a}$ & $P_{z} \quad \sin \theta$ & $\cos \theta_{a}$ & $\sin 2 \theta_{b}$ & $\sin \phi_{b}$ \\
\hline-6 & $\operatorname{Im}\left\{A_{+}^{*} A_{-}\right\}$ & $\alpha_{a}$ & $P_{z} \quad \sin \theta$ & $\sin \theta_{a}$ & $\cos ^{2} \theta_{b}$ & $\sin \phi_{a}$ \\
\hline+3 & $\operatorname{Im}\left\{B_{+}^{*} B_{-}\right\}$ & $\alpha_{a}$ & $P_{z} \quad \sin \theta$ & $\sin \theta_{a}$ & $\sin ^{2} \theta_{b}$ & $\sin \left(\phi_{a}+2 \phi_{b}\right)$ \\
\hline$+3 / \sqrt{2}$ & $\operatorname{Im}\left\{A_{+}^{*} B_{-}\right\}+\operatorname{Im}\left\{A_{-}^{*} B_{+}\right\}$ & $\alpha_{a}$ & & $\sin \theta_{a}$ & $\sin 2 \theta_{b}$ & $\sin \left(\phi_{a}+\phi_{b}\right)$ \\
\hline
\end{tabular}

Table 3. Various contributions to the angular distribution of the $0^{1 / 2} \rightarrow a^{1 / 2} b^{1} \rightarrow 1^{1 / 2} 2^{0} 3^{0} 4^{0}$ process, with conventions and assumptions specified in the text. Each line corresponds to a term of different angular dependence (most of them being independent). The separation in columns is only meant to ease the comparison between the various factors appearing in each term. The four blocks distinguish terms whose combinations of angular and polarisation dependence have different parity and motion reversal transformation properties. They are respectively P-even- $\hat{T}$-even, P-odd- $\hat{T}$-even, P-even- $\hat{T}$-odd, and P-odd- $\hat{\mathrm{T}}$-odd. 


\begin{tabular}{|c|c|c|c|c|c|}
\hline$+3 / 4$ & $\left|B_{+}\right|^{2}+\left|B_{-}\right|^{2}+3\left|C_{+}\right|^{2}+3\left|C_{-}\right|^{2}$ & & & $\sin ^{2} \theta_{b}$ & \\
\hline$+3 \sqrt{2 / 3} / 2$ & $\operatorname{Re}\left\{A_{+}^{*} C_{+}\right\}+\operatorname{Re}\left\{A_{-}^{*} C_{-}\right\}$ & & $\sin 2 \theta_{a}$ & $\sin 2 \theta_{b}$ & $\cos \left(\phi_{a}+\phi_{b}\right)$ \\
\hline$+3 / 2$ & $\left|A_{+}\right|^{2}+\left|A_{-}\right|^{2}$ & & $\left(1+3 \cos ^{2} \theta_{a}\right)$ & $\cos ^{2} \theta_{b}$ & \\
\hline$+3 \sqrt{3} / 2$ & $\operatorname{Re}\left\{B_{+}^{*} C_{-}\right\}+\operatorname{Re}\left\{B_{-}^{*} C_{+}\right\}$ & & $\sin ^{2} \theta_{a}$ & $\sin ^{2} \theta_{b}$ & $\cos \left(2 \phi_{a}+2 \phi_{b}\right)$ \\
\hline$+9 / 4$ & $\left|B_{+}\right|^{2}+\left|B_{-}\right|^{2}-\left|C_{+}\right|^{2}-\left|C_{-}\right|^{2}$ & & $\cos ^{2} \theta_{a}$ & $\sin ^{2} \theta_{b}$ & \\
\hline$-3 / 4$ & $5\left|B_{+}\right|^{2}-5\left|B_{-}\right|^{2}-3\left|C_{+}\right|^{2}+3\left|C_{-}\right|^{2}$ & $\alpha_{a}$ & $\cos \theta_{a}$ & $\sin ^{2} \theta_{b}$ & \\
\hline$-3 / 2$ & $\left|A_{+}\right|^{2}-\left|A_{-}\right|^{2}$ & $\alpha_{a}$ & $\left(5-9 \cos ^{2} \theta_{a}\right) \cos \theta_{a}$ & $\cos ^{2} \theta_{b}$ & \\
\hline$-3 \sqrt{2 / 3} / 2$ & $\operatorname{Re}\left\{A_{+}^{*} C_{+}\right\}-\operatorname{Re}\left\{A_{-}^{*} C_{-}\right\}$ & $\alpha_{a}$ & $\left(1-3 \cos ^{2} \theta_{a}\right) \sin \theta_{a}$ & $\sin 2 \theta_{b}$ & $\cos \left(\phi_{a}+\phi_{b}\right)$ \\
\hline$-3 / 2 \sqrt{2}$ & $\operatorname{Re}\left\{A_{+}^{*} B_{-}\right\}-\operatorname{Re}\left\{A_{-}^{*} B_{+}\right\}$ & $\alpha_{a}$ & $\left(1-9 \cos ^{2} \theta_{a}\right) \sin \theta_{a}$ & $\sin 2 \theta_{b}$ & $\cos \left(\phi_{a}+\phi_{b}\right)$ \\
\hline$-9 \sqrt{3} / 2$ & $\operatorname{Re}\left\{B_{+}^{*} C_{-}\right\}-\operatorname{Re}\left\{B_{-}^{*} C_{+}\right\}$ & $\alpha_{a}$ & $\sin ^{2} \theta_{a} \cos \theta_{a}$ & $\sin ^{2} \theta_{b}$ & $\cos \left(2 \phi_{a}+2 \phi_{b}\right)$ \\
\hline$+9 / 4$ & $3\left|B_{+}\right|^{2}-3\left|B_{-}\right|^{2}-\left|C_{+}\right|^{2}+\left|C_{-}\right|^{2}$ & $\alpha_{a}$ & $\cos ^{3} \theta_{a}$ & $\sin ^{2} \theta_{b}$ & \\
\hline$-3 / 4$ & $\left|B_{+}\right|^{2}-\left|B_{-}\right|^{2}-3\left|C_{+}\right|^{2}+3\left|C_{-}\right|^{2}$ & $P_{z} \cos \theta$ & & $\sin ^{2} \theta_{b}$ & \\
\hline$+3 \sqrt{2 / 3} / 2$ & $\operatorname{Re}\left\{A_{+}^{*} C_{+}\right\}-\operatorname{Re}\left\{A_{-}^{*} C_{-}\right\}$ & $P_{z} \cos \theta$ & $\sin 2 \theta_{a}$ & $\sin 2 \theta_{b}$ & $\cos \left(\phi_{a}+\phi_{b}\right)$ \\
\hline$+3 / 2$ & $\left|A_{+}\right|^{2}-\left|A_{-}\right|^{2}$ & $P_{z} \cos \theta$ & $\left(1+3 \cos ^{2} \theta_{a}\right)$ & $\cos ^{2} \theta_{b}$ & \\
\hline$-3 \sqrt{3} / 2$ & $\operatorname{Re}\left\{B_{+}^{*} C_{-}\right\}-\operatorname{Re}\left\{B_{-}^{*} C_{+}\right\}$ & $P_{z} \cos \theta$ & $\sin ^{2} \theta_{a}$ & $\sin ^{2} \theta_{b}$ & $\cos \left(2 \phi_{a}+2 \phi_{b}\right)$ \\
\hline$-9 / 4$ & $\left|B_{+}\right|^{2}-\left|B_{-}\right|^{2}+\left|C_{+}\right|^{2}-\left|C_{-}\right|^{2}$ & $P_{z} \cos \theta$ & $\cos ^{2} \theta_{a}$ & $\sin ^{2} \theta_{b}$ & \\
\hline$+3 \sqrt{3} / 2$ & $\operatorname{Re}\left\{B_{+}^{*} C_{+}\right\}-\operatorname{Re}\left\{B_{-}^{*} C_{-}\right\}$ & $P_{z} \sin \theta$ & $\sin 2 \theta_{a}$ & $\sin ^{2} \theta_{b}$ & $\cos \phi_{a}$ \\
\hline$+3 / 2 \sqrt{2}$ & $\operatorname{Re}\left\{A_{+}^{*} B_{+}\right\}-\operatorname{Re}\left\{A_{-}^{*} B_{-}\right\}$ & $P_{z} \sin \theta$ & $\left(1+3 \cos ^{2} \theta_{a}\right)$ & $\sin 2 \theta_{b}$ & $\cos \phi_{b}$ \\
\hline$+3 \sqrt{2 / 3} / 2$ & $\operatorname{Re}\left\{A_{+}^{*} C_{-}\right\}-\operatorname{Re}\left\{A_{-}^{*} C_{+}\right\}$ & $P_{z} \sin \theta$ & $\sin ^{2} \theta_{a}$ & $\sin 2 \theta_{b}$ & $\cos \left(2 \phi_{a}+\phi_{b}\right)$ \\
\hline$+3 / 4$ & $5\left|B_{+}\right|^{2}+5\left|B_{-}\right|^{2}+3\left|C_{+}\right|^{2}+3\left|C_{-}\right|^{2}$ & $\alpha_{a} P_{z} \cos \theta$ & $\cos \theta_{a}$ & $\sin ^{2} \theta_{b}$ & \\
\hline$-3 / 2$ & $\left|A_{+}\right|^{2}+\left|A_{-}\right|^{2}$ & $\alpha_{a} P_{z} \cos \theta$ & $\left(5-9 \cos ^{2} \theta_{a}\right) \cos \theta_{a}$ & $\cos ^{2} \theta_{b}$ & \\
\hline$-3 \sqrt{2 / 3} / 2$ & $\operatorname{Re}\left\{A_{+}^{*} C_{+}\right\}+\operatorname{Re}\left\{A_{-}^{*} C_{-}\right\}$ & $\alpha_{a} P_{z} \cos \theta$ & $\left(1-3 \cos ^{2} \theta_{a}\right) \sin \theta_{a}$ & $\sin 2 \theta_{b}$ & $\cos \left(\phi_{a}+\phi_{b}\right)$ \\
\hline$-3 / 2 \sqrt{2}$ & $\operatorname{Re}\left\{A_{+}^{*} B_{-}\right\}+\operatorname{Re}\left\{A_{-}^{*} B_{+}\right\}$ & $\alpha_{a} P_{z} \cos \theta$ & $\left(1-9 \cos ^{2} \theta_{a}\right) \sin \theta_{a}$ & $\sin 2 \theta_{b}$ & $\cos (\phi$ \\
\hline$+9 \sqrt{3} / 2$ & $\operatorname{Re}\left\{B_{+}^{*} C_{-}\right\}+\operatorname{Re}\left\{B_{-}^{*} C_{+}\right\}$ & $\alpha_{a} P_{z} \cos \theta$ & $\sin ^{2} \theta_{a} \cos \theta_{a}$ & $\sin ^{2} \theta_{b}$ & $\cos \left(2 \phi_{a}+2 \phi_{b}\right)$ \\
\hline$-9 / 4$ & $3\left|B_{+}\right|^{2}+3\left|B_{-}\right|^{2}+\left|C_{+}\right|^{2}+\left|C_{-}\right|^{2}$ & $\alpha_{a} P_{z} \cos \theta$ & $\cos ^{3} \theta_{a}$ & $\sin ^{2} \theta_{b}$ & \\
\hline$-3 / 2 \sqrt{2}$ & $\operatorname{Re}\left\{A_{+}^{*} B_{+}\right\}+\operatorname{Re}\left\{A_{-}^{*} B_{-}\right\}$ & $\alpha_{a} P_{z} \sin \theta$ & $\left(5-9 \cos ^{2} \theta_{a}\right) \cos \theta_{a}$ & $\sin 2 \theta_{b}$ & $\cos \phi_{b}$ \\
\hline$-3 \sqrt{3} / 2$ & $\operatorname{Re}\left\{B_{+}^{*} C_{+}\right\}+\operatorname{Re}\left\{B_{-}^{*} C_{-}\right\}$ & $\alpha_{a} P_{z} \sin \theta$ & $\left(1-3 \cos ^{2} \theta_{a}\right) \sin \theta_{a}$ & $\sin ^{2} \theta_{b}$ & $\cos \phi_{a}$ \\
\hline+3 & $\operatorname{Re}\left\{A_{+}^{*} A_{-}\right\}$ & $\alpha_{a} P_{z} \sin \theta$ & $\left(1-9 \cos ^{2} \theta_{a}\right) \sin \theta_{a}$ & $\cos ^{2} \theta_{b}$ & $\cos \phi_{a}$ \\
\hline$-3 / 2$ & $\operatorname{Re}\left\{B_{+}^{*} B_{-}\right\}$ & $\alpha_{a} P_{z} \sin \theta$ & $\left(1-9 \cos ^{2} \theta_{a}\right) \sin \theta_{a}$ & $\sin ^{2} \theta_{b}$ & $\cos \left(\phi_{a}+2 \phi_{b}\right)$ \\
\hline$-9 / 2$ & $\operatorname{Re}\left\{C_{+}^{*} C_{-}\right\}$ & $\alpha_{a} P_{z} \sin \theta$ & $\sin ^{3} \theta_{a}$ & $\sin ^{2} \theta_{b}$ & $\cos \left(3 \phi_{a}+2 \phi_{b}\right)$ \\
\hline$-9 \sqrt{2 / 3} / 2$ & $\operatorname{Re}\left\{A_{+}^{*} C_{-}\right\}+\operatorname{Re}\left\{A_{-}^{*} C_{+}\right\}$ & $\alpha_{a} P_{z} \sin \theta$ & $\sin ^{2} \theta_{a} \cos \theta_{a}$ & $\sin 2 \theta_{b}$ & $\cos \left(2 \phi_{a}+\phi_{b}\right)$ \\
\hline$-3 \sqrt{2 / 3} / 2$ & $\operatorname{Im}\left\{A_{+}^{*} C_{+}\right\}+\operatorname{Im}\left\{A_{-}^{*} C_{-}\right\}$ & $P_{z} \cos \theta$ & $\sin 2 \theta_{a}$ & $\sin 2 \theta_{b}$ & $\sin \left(\phi_{a}+\phi_{b}\right)$ \\
\hline$-3 \sqrt{3} / 2$ & $\operatorname{Im}\left\{B_{+}^{*} C_{-}\right\}+\operatorname{Im}\left\{B_{-}^{*} C_{+}\right\}$ & $P_{z} \cos \theta$ & $\sin ^{2} \theta_{a}$ & $\sin ^{2} \theta_{b}$ & $\sin \left(2 \phi_{a}+2 \phi_{b}\right)$ \\
\hline$-3 \sqrt{3} / 2$ & $\operatorname{Im}\left\{B_{+}^{*} C_{+}\right\}+\operatorname{Im}\left\{B_{-}^{*} C_{-}\right\}$ & $P_{z} \sin \theta$ & $\sin 2 \theta_{a}$ & $\sin ^{2} \theta_{b}$ & $\sin \phi_{a}$ \\
\hline$-3 / 2 \sqrt{2}$ & $\operatorname{Im}\left\{A_{+}^{*} B_{+}\right\}+\operatorname{Im}\left\{A_{-}^{*} B_{-}\right\}$ & $P_{z} \sin \theta$ & $\left(1+3 \cos ^{2} \theta_{a}\right)$ & $\sin 2 \theta_{b}$ & $\sin \phi_{b}$ \\
\hline$+3 \sqrt{2 / 3} / 2$ & $\operatorname{Im}\left\{A_{+}^{*} C_{-}\right\}+\operatorname{Im}\left\{A_{-}^{*} C_{+}\right\}$ & $P_{z} \sin \theta$ & $\sin ^{2} \theta_{a}$ & $\sin 2 \theta_{b}$ & $\sin \left(2 \phi_{a}+\phi_{b}\right)$ \\
\hline$+3 \sqrt{2 / 3} / 2$ & $\operatorname{Im}\left\{A_{+}^{*} C_{+}\right\}-\operatorname{Im}\left\{A_{-}^{*} C_{-}\right\}$ & $\alpha_{a} P_{z} \cos \theta$ & $\left(1-3 \cos ^{2} \theta_{a}\right) \sin \theta_{a}$ & $\sin 2 \theta_{b}$ & $\sin \left(\phi_{a}+\phi_{b}\right)$ \\
\hline$-3 / 2 \sqrt{2}$ & $\operatorname{Im}\left\{A_{+}^{*} B_{-}\right\}-\operatorname{Im}\left\{A_{-}^{*} B_{+}\right\}$ & $\alpha_{a} P_{z} \cos \theta$ & $\left(1-9 \cos ^{2} \theta_{a}\right) \sin \theta_{a}$ & $\sin 2 \theta_{b}$ & $\sin \left(\phi_{a}+\phi_{b}\right)$ \\
\hline$+9 \sqrt{3} / 2$ & $\operatorname{Im}\left\{B_{+}^{*} C_{-}\right\}-\operatorname{Im}\left\{B_{-}^{*} C_{+}\right\}$ & $\alpha_{a} P_{z} \cos \theta$ & $\sin ^{2} \theta_{a} \cos \theta_{a}$ & $\sin ^{2} \theta_{b}$ & $\sin \left(2 \phi_{a}+2 \phi_{b}\right)$ \\
\hline$+3 / 2 \sqrt{2}$ & $\operatorname{Im}\left\{A_{+}^{*} B_{+}\right\}-\operatorname{Im}\left\{A_{-}^{*} B_{-}\right\}$ & $\alpha_{a} P_{z} \sin \theta$ & $\left(5-9 \cos ^{2} \theta_{a}\right) \cos \theta_{a}$ & $\sin 2 \theta_{b}$ & $\sin \phi_{b}$ \\
\hline$+3 \sqrt{3} / 2$ & $\operatorname{Im}\left\{B_{+}^{*} C_{+}\right\}-\operatorname{Im}\left\{B_{-}^{*} C_{-}\right\}$ & $\alpha_{a} P_{z} \sin \theta$ & $\left(1-3 \cos ^{2} \theta_{a}\right) \sin \theta_{a}$ & $\sin ^{2} \theta_{b}$ & $\sin \phi_{a}$ \\
\hline+3 & $\operatorname{Im}\left\{A_{+}^{*} A_{-}\right\}$ & $\alpha_{a} P_{z} \sin \theta$ & $\left(1-9 \cos ^{2} \theta_{a}\right) \sin \theta_{a}$ & $\cos ^{2} \theta_{b}$ & $\sin \phi_{a}$ \\
\hline$-3 / 2$ & $\operatorname{Im}\left\{B_{+}^{*} B_{-}\right\}$ & $\alpha_{a} P_{z} \sin \theta$ & $\left(1-9 \cos ^{2} \theta_{a}\right) \sin \theta_{a}$ & $\sin ^{2} \theta_{b}$ & $\sin \left(\phi_{a}+2 \phi_{b}\right)$ \\
\hline$-9 / 2$ & $\operatorname{Im}\left\{C_{+}^{*} C_{-}\right\}$ & $\alpha_{a} P_{z} \sin \theta$ & $\sin ^{3} \theta_{a}$ & $\sin ^{2} \theta_{b}$ & $\sin \left(3 \phi_{a}+2 \phi_{b}\right)$ \\
\hline$-9 \sqrt{2 / 3} / 2$ & $\operatorname{Im}\left\{A_{+}^{*} C_{-}\right\}-\operatorname{Im}\left\{A_{-}^{*} C_{+}\right\}$ & $\alpha_{a} P_{z} \sin \theta$ & $\sin ^{2} \theta_{a} \cos \theta_{a}$ & $\sin 2 \theta_{b}$ & $\sin \left(2 \phi_{a}+\phi_{b}\right)$ \\
\hline$-3 \sqrt{2 / 3} / 2$ & $\operatorname{Im}\left\{A_{+}^{*} C_{+}\right\}-\operatorname{Im}\left\{A_{-}^{*} C_{-}\right\}$ & & $\sin 2 \theta_{a}$ & $\sin 2 \theta_{b}$ & $\sin \left(\phi_{a}+\phi_{b}\right)$ \\
\hline$+3 \sqrt{3} / 2$ & $\operatorname{Im}\left\{B_{+}^{*} C_{-}\right\}-\operatorname{Im}\left\{B_{-}^{*} C_{+}\right\}$ & & $\sin ^{2} \theta_{a}$ & $\sin ^{2} \theta_{b}$ & $\sin \left(2 \phi_{a}+2 \phi_{b}\right)$ \\
\hline$+3 \sqrt{2 / 3} / 2$ & $\operatorname{Im}\left\{A_{+}^{*} C_{+}\right\}+\operatorname{Im}\left\{A_{-}^{*} C_{-}\right\}$ & $\alpha_{a}$ & $\left(1-3 \cos ^{2} \theta_{a}\right) \sin \theta_{a}$ & $\sin 2 \theta_{b}$ & $\sin \left(\phi_{a}+\phi_{b}\right)$ \\
\hline$-3 / 2 \sqrt{2}$ & $\operatorname{Im}\left\{A_{+}^{*} B_{-}\right\}+\operatorname{Im}\left\{A_{-}^{*} B_{+}\right\}$ & $\alpha_{a}$ & $\left(1-9 \cos ^{2} \theta_{a}\right) \sin \theta_{a}$ & $\sin 2 \theta_{b}$ & $\sin \left(\phi_{a}+\phi_{b}\right)$ \\
\hline$-9 \sqrt{3} / 2$ & $\operatorname{Im}\left\{B_{+}^{*} C_{-}\right\}+\operatorname{Im}\left\{B_{-}^{*} C_{+}\right\}$ & $\alpha_{a}$ & $\sin ^{2} \theta_{a} \cos \theta_{a}$ & $\sin ^{2} \theta_{b}$ & $\sin \left(2 \phi_{a}+2 \phi_{b}\right)$ \\
\hline
\end{tabular}

Table 4. Same as table 3 , for the $0^{1 / 2} \rightarrow a^{3 / 2} b^{1} \rightarrow 1^{1 / 2} 2^{0} 3^{0} 4^{0}$ process, where particle $a$ has spin $3 / 2$ instead of $1 / 2$. 


$$
\begin{array}{|llllll|}
\hline+\left|B_{+}\right|^{2}+\left|B_{-}\right|^{2} & & & & \\
+ & \left|B_{+}\right|^{2}-\left|B_{-}\right|^{2} & \alpha_{a} & & & \cos \theta_{a} \\
\hline- & \left|B_{+}\right|^{2}-\left|B_{-}\right|^{2} & & P_{z} & \cos \theta & \\
- & \left|B_{+}\right|^{2}+\left|B_{-}\right|^{2} & \alpha_{a} & P_{z} & \cos \theta & \cos \theta_{a} \\
\hline
\end{array}
$$

Table 5. Contributions to the angular distribution of the three-body $0^{1 / 2} \rightarrow a^{1 / 2} b^{1} \rightarrow 1^{1 / 2} 2^{0} b^{1}$ decay, under the conventions and assumptions specified in the text. The four blocks distinguish terms whose combinations of angular and polarisation dependence are respectively P-even- $\hat{T}$-even and P-odd- $\hat{T}$-even. A third block, which receives no contributions here, includes P-even- $\hat{\mathrm{T}}$-odd decay terms in the subsequent tables of this series.

\begin{tabular}{|lcccccc|}
\hline$+1 / 2$ & $\left|B_{+}\right|^{2}+\left|B_{-}\right|^{2}$ & & & $\left(1+3 \cos ^{2} \theta_{a}\right)$ & \\
$+3 / 2$ & $\left|C_{+}\right|^{2}+\left|C_{-}\right|^{2}$ & & & $\sin ^{2} \theta_{a}$ & \\
$-1 / 2$ & $\left|B_{+}\right|^{2}-\left|B_{-}\right|^{2}$ & $\alpha_{a}$ & & & $\left(5-9 \cos ^{2} \theta_{a}\right) \cos \theta_{a}$ & \\
$+3 / 4$ & $\left|C_{+}\right|^{2}-\left|C_{-}\right|^{2}$ & $\alpha_{a}$ & & & $\sin \theta_{a} \sin 2 \theta_{a}$ & \\
\hline$+\sqrt{3}$ & $\operatorname{Re}\left\{B_{+}^{*} C_{+}\right\}-\operatorname{Re}\left\{B_{-}^{*} C_{-}\right\}$ & & $P_{z}$ & $\sin \theta$ & $\sin 2 \theta_{a}$ & $\cos \phi_{a}$ \\
$-1 / 2$ & $\left|B_{+}\right|^{2}-\left|B_{-}\right|^{2}$ & & $P_{z}$ & $\cos \theta$ & $\left(1+3 \cos ^{2} \theta_{a}\right)$ & \\
$+3 / 2$ & $\left|C_{+}\right|^{2}-\left|C_{-}\right|^{2}$ & & $P_{z}$ & $\cos \theta$ & $\sin \theta_{a}$ & \\
$-\sqrt{3}$ & $\operatorname{Re}\left\{B_{+}^{*} C_{+}\right\}+\operatorname{Re}\left\{B_{-}^{*} C_{-}\right\}$ & $\alpha_{a}$ & $P_{z}$ & $\sin \theta$ & $\left(1-3 \cos ^{2} \theta_{a}\right) \sin \theta_{a}$ & $\cos \phi_{a}$ \\
$+1 / 2$ & $\left|B_{+}\right|^{2}+\left|B_{-}\right|^{2}$ & $\alpha_{a}$ & $P_{z}$ & $\cos \theta$ & $\left(5-9 \cos ^{2} \theta_{a}\right) \cos \theta_{a}$ & \\
$+3 / 4$ & $\left|C_{+}\right|^{2}+\left|C_{-}\right|^{2}$ & $\alpha_{a}$ & $P_{z}$ & $\cos \theta$ & $\sin \theta_{a} \sin 2 \theta_{a}$ & \\
\hline$-\sqrt{3}$ & $\operatorname{Im}\left\{B_{+}^{*} C_{+}\right\}+\operatorname{Im}\left\{B_{-}^{*} C_{-}\right\}$ & & $P_{z}$ & $\sin \theta$ & $\sin 2 \theta_{a}$ & $\sin \phi_{a}$ \\
$+\sqrt{3}$ & $\operatorname{Im}\left\{B_{+}^{*} C_{+}\right\}-\operatorname{Im}\left\{B_{-}^{*} C_{-}\right\}$ & $\alpha_{a}$ & $P_{z}$ & $\sin \theta$ & $\left(1-3 \cos ^{2} \theta_{a}\right) \sin \theta_{a}$ & $\sin \phi_{a}$ \\
\hline
\end{tabular}

Table 6. Same as table 5 , for the $0^{1 / 2} \rightarrow a^{3 / 2} b^{1} \rightarrow 1^{1 / 2} 2^{0} b^{1}$ process, where particle $a$ has spin $3 / 2$ instead of $1 / 2$. 


\begin{tabular}{|lllllll|}
\hline+ & $\left|A_{+}\right|^{2}+\left|A_{-}\right|^{2}$ & & & & & \\
+ & $\left|A_{+}\right|^{2}-\left|A_{-}\right|^{2}$ & $\alpha_{a}$ & \multicolumn{4}{c|}{$\cos \theta_{a}$} \\
\hline+ & $\left|A_{+}\right|^{2}-\left|A_{-}\right|^{2}$ & & $P_{z}$ & $\cos \theta$ & & \\
-2 & $\operatorname{Re}\left\{A_{+}^{*} A_{-}\right\}$ & $\alpha_{a}$ & $P_{z}$ & $\sin \theta$ & $\sin \theta_{a}$ & $\cos \phi_{a}$ \\
+ & $\left|A_{+}\right|^{2}+\left|A_{-}\right|^{2}$ & $\alpha_{a}$ & $P_{z}$ & $\cos \theta$ & $\cos \theta_{a}$ & \\
\hline-2 & $\operatorname{Im}\left\{A_{+}^{*} A_{-}\right\}$ & $\alpha_{a}$ & $P_{z}$ & $\sin \theta$ & $\sin \theta_{a}$ & $\sin \phi_{a}$ \\
\hline
\end{tabular}

Table 7. Same as table 5 , for the $0^{1 / 2} \rightarrow a^{1 / 2} b^{0} \rightarrow 1^{1 / 2} 2^{0} b^{0}$ process, where particle $b$ has spin 0 instead of 1 .

\begin{tabular}{|ccccccc|}
\hline$+1 / 2$ & $\left|A_{+}\right|^{2}+\left|A_{-}\right|^{2}$ & & & & $\left(1+3 \cos ^{2} \theta_{a}\right)$ & \\
$-1 / 2$ & $\left|A_{+}\right|^{2}-\left|A_{-}\right|^{2}$ & $\alpha_{a}$ & & & $\left(5-9 \cos ^{2} \theta_{a}\right) \cos \theta_{a}$ & \\
\hline$+1 / 2$ & $\left|A_{+}\right|^{2}-\left|A_{-}\right|^{2}$ & & $P_{z}$ & $\cos \theta$ & $\left(1+3 \cos ^{2} \theta_{a}\right)$ & \\
+ & $\operatorname{Re}\left\{A_{+}^{*} A_{-}\right\}$ & $\alpha_{a}$ & $P_{z}$ & $\sin \theta$ & $\left(1-9 \cos ^{2} \theta_{a}\right) \sin \theta_{a}$ & $\cos \phi_{a}$ \\
$-1 / 2$ & $\left|A_{+}\right|^{2}+\left|A_{-}\right|^{2}$ & $\alpha_{a}$ & $P_{z}$ & $\cos \theta$ & $\left(5-9 \cos ^{2} \theta_{a}\right) \cos \theta_{a}$ & \\
\hline+ & $\operatorname{Im}\left\{A_{+}^{*} A_{-}\right\}$ & $\alpha_{a}$ & $P_{z}$ & $\sin \theta$ & $\left(1-9 \cos ^{2} \theta_{a}\right) \sin \theta_{a}$ & $\sin \phi_{a}$ \\
\hline
\end{tabular}

Table 8. Same as table 7 , for the $0^{1 / 2} \rightarrow a^{3 / 2} b^{0} \rightarrow 1^{1 / 2} 2^{0} b^{0}$ process, where particle $a$ has spin $3 / 2$ instead of $1 / 2$.

where $\boldsymbol{p}_{\mathbf{3}}$ and $\boldsymbol{p}_{\boldsymbol{4}}$ are the momenta of particles 3 and 4 in particle 0 's restframe, one can further write

$$
\left(0=\boldsymbol{n}_{\boldsymbol{a}} \cdot \boldsymbol{z}_{\boldsymbol{a}},\right) \quad \cos \phi_{a}=-\boldsymbol{n}_{\boldsymbol{a}} \cdot \boldsymbol{y}_{\boldsymbol{a}}, \quad \sin \phi_{a}=\boldsymbol{n}_{\boldsymbol{a}} \cdot \boldsymbol{x}_{\boldsymbol{a}}
$$

and similarly for $a \leftrightarrow b$. This shows that $\cos \phi_{a}$ and $\cos \phi_{b}$ are P-odd- $\hat{\mathrm{T}}$-odd variables, while $\sin \phi_{a}$ and $\sin \phi_{b}$ are P-even- $\hat{T}$-even. With $\tilde{\boldsymbol{p}}_{\mathbf{1}}$ and $\tilde{\boldsymbol{p}}_{\mathbf{3}}$, the momenta of particle 1 and 3 , respectively measured in the particle $a$ and $b$ restframes, one can define

$$
\cos \theta_{a}=\boldsymbol{z}_{\boldsymbol{a}} \cdot \frac{\tilde{\boldsymbol{p}}_{\mathbf{1}}}{\left|\tilde{\boldsymbol{p}}_{\mathbf{1}}\right|}, \quad \sin \theta_{a}=+\sqrt{1-\cos ^{2} \theta_{a}},
$$

and similarly for $a \leftrightarrow b$ and $1 \leftrightarrow 3$, demonstrating that both $\cos \theta_{a, b}$ and $\sin \theta_{a, b}$ are P-even$\hat{\mathrm{T}}$-even variables. Finally, the P-odd- $\hat{\mathrm{T}}$-odd character of the $\boldsymbol{z}$ vector implies that among the polarisations components

$$
P_{x}=\langle\boldsymbol{s} \cdot \boldsymbol{x}\rangle, \quad P_{y}=\langle\boldsymbol{s} \cdot \boldsymbol{y}\rangle, \quad P_{z}=\langle\boldsymbol{s} \cdot \boldsymbol{z}\rangle,
$$


$P_{x}$ and $P_{y}$ are $\mathrm{P}$-odd- $\hat{\mathrm{T}}$-even, while $P_{z}$ is $\mathrm{P}$-even- $\hat{\mathrm{T}}$-odd. Their values are fixed by particle 0 's production amplitudes and, in general, depend on the production kinematics which is disregarded here.

To summarize, for our definition of frames, we have thus identified three P-odd- $\hat{\mathrm{T}}$-odd kinematic variables:

$$
\cos \theta, \quad \cos \phi_{a}, \quad \text { and } \cos \phi_{b},
$$

while $\cos \phi, \sin \phi, \cos \theta_{a, b}, \sin \phi_{a, b}$, as well as $m_{a, b}$ which are necessary to fully specify the final-state kinematics, are all P-even- $\hat{T}$-even. The contributions to the angular distributions we displayed in table $1-8$ have been grouped according to their $\mathrm{P}$ and $\hat{\mathrm{T}}$ transformation properties. In table 1-4 relating to four-body decays, the angular distributions of the contributions in the first and third blocks are P-even- $\hat{\mathrm{T}}$-even while that of the second and fourth ones are P-odd- $\hat{T}$-odd. The second and third blocks moreover include contributions proportional to the P-even- $\hat{\mathrm{T}}$-odd polarisation $P_{z}$. As a result, the four blocks distinguish contributions whose combinations of angular and polarisation dependence are respectively P-even- $\hat{T}$-even, P-odd- $\hat{T}$-even, P-even- $\hat{\mathrm{T}}$-odd, and P-odd- $\hat{\mathrm{T}}$-odd. In three-body decays, there are not enough independent four-momenta to form $\hat{\mathrm{T}}$-odd $\epsilon_{\mu \nu \rho \sigma} p_{1}^{\mu} p_{2}^{\nu} p_{3}^{\rho} p_{4}^{\sigma}$ contractions. One must necessarily involve a spin four-vector. In table $5-8$, all terms proportional to imaginary parts of decay amplitude interferences therefore come proportional to $P_{z}$. They thus appear in P-even- $\hat{\mathrm{T}}$-odd blocks, and there are no fourth P-odd- $\hat{\mathrm{T}}$-odd ones. The $0^{1 / 2} \rightarrow a^{1 / 2} b^{1} \rightarrow 1^{1 / 2} 2^{0} b^{1}$ decay relating to table 5 does moreover not contain any term proportional to the imaginary part of decay amplitude interferences when $b^{1}$ is massless (an assumption relaxed in appendix A).

\section{Asymmetries}

As mentioned in the introduction, due to the presence of a $\hat{\mathrm{T}}$-odd polarisation component $P_{z}$, both $\hat{\mathrm{T}}$-odd and $\hat{\mathrm{T}}$-even angular asymmetries can potentially serve to access imaginary parts of decay amplitude interferences.

\section{1 $\hat{\mathbf{T}}$-odd angular asymmetries}

In the spirit of ref. [2], $\hat{\mathrm{T}}$-odd-CP-odd angular asymmetries could be constructed systematically as

$$
\mathcal{A}_{m n o}^{j k l} \equiv \int \mathrm{d} \Omega\left(\frac{1}{\Gamma} \frac{\mathrm{d} \Gamma}{\mathrm{d} \Omega}-\frac{1}{\bar{\Gamma}} \frac{\mathrm{d} \bar{\Gamma}}{\mathrm{d} \Omega}\right) \operatorname{sign}\left\{f_{j}(\cos \theta) f_{k}\left(\cos \theta_{a}\right) f_{l}\left(\cos \theta_{b}\right) \sin \left(m \phi_{a}+n \phi_{b}+o \frac{\pi}{2}\right)\right\}
$$

for $f_{0}(x)=1, f_{1}(x)=x, f_{2}(x)=3 x^{2}-1$, etc. which could be chosen as Legendre polynomials and various $j, k, l, m, n, o$ combinations of integers satisfying $j+m+n+o \in 2 \mathbb{Z}$ with $o \in\{0,1\}$. Contributions not explicitly listed in the various tables of this paper could appear in the interferences of amplitudes featuring $a$ and $b$ intermediate states of various spins or different topologies. It was also noted in ref. [2] that distinguishing regions in the $m_{a, b}$ invariant mass integration could be useful when resonances are identified, and that pairings of final-state particles different from the $a=(12), b=(34)$ ones could increase the 
sensitivity to phase differences between amplitudes of different resonance structures. Understanding the origin of the various angular distribution components is however required to determine whether a symmetry violation observed arises from the decay or production, given the lack of decoupling between the two parts of the process.

This understanding we gained in the previous section allows us to be more specific. In both four-body processes featuring a spin- $1 / 2$ intermediate resonance $a$, under the assumptions stated, there is actually one single $\hat{\mathrm{T}}$-odd angular distribution that provides access to $\mathrm{CP}$-odd phase differences between decay amplitudes, without requiring $\mathrm{CP}$-even phase between neither decay nor production amplitudes. The corresponding term is displayed in the fourth blocks of tables 1 and 3. By relying on the

$$
\frac{1}{\Gamma} \int \mathrm{d} \Omega \frac{\mathrm{d} \Gamma}{\mathrm{d} \Omega} \operatorname{sign}\left\{\cos \theta_{b} \sin \left(\phi_{a}+\phi_{b}\right)\right\}
$$

asymmetry, or on the analogue moment, one gets access to the $\left(\operatorname{Im}\left\{A_{+}^{*} B_{-}\right\}+\right.$ $\left.\operatorname{Im}\left\{A_{-}^{*} B_{+}\right\}\right) \alpha_{a}$ combination of decay amplitudes. Then combining the CP-conjugate $0 \rightarrow 1234$ and $\overline{0} \rightarrow \overline{1} \overline{2} \overline{3} \overline{4}$ processes to form $\mathcal{A}_{110}^{001}$ yields sensitivity to small differences in the CP-odd phases between the $A_{+} B_{-}$or $A_{-} B_{+}$amplitudes. It is maximal when they have identical CP-even phases. In both four-body processes featuring a spin $3 / 2$ intermediate resonance $a$, one could moreover employ on the

$$
\begin{aligned}
& \mathcal{A}_{110}^{011}=\int \mathrm{d} \Omega\left(\frac{1}{\Gamma} \frac{\mathrm{d} \Gamma}{\mathrm{d} \Omega}-\frac{1}{\bar{\Gamma}} \frac{\mathrm{d} \bar{\Gamma}}{\mathrm{d} \Omega}\right) \operatorname{sign}\left\{\cos \theta_{a} \cos \theta_{b} \sin \left(\phi_{a}+\phi_{b}\right)\right\} \\
& \mathcal{A}_{220}^{000}=\int \mathrm{d} \Omega\left(\frac{1}{\Gamma} \frac{\mathrm{d} \Gamma}{\mathrm{d} \Omega}-\frac{1}{\bar{\Gamma}} \frac{\mathrm{d} \bar{\Gamma}}{\mathrm{d} \Omega}\right) \operatorname{sign}\left\{\sin \left(2 \phi_{a}+2 \phi_{b}\right)\right\} \\
& \mathcal{A}_{110}^{021}=\int \mathrm{d} \Omega\left(\frac{1}{\Gamma} \frac{\mathrm{d} \Gamma}{\mathrm{d} \Omega}-\frac{1}{\bar{\Gamma}} \frac{\mathrm{d} \bar{\Gamma}}{\mathrm{d} \Omega}\right) \operatorname{sign}\left\{\left(3 \cos ^{2} \theta_{a}-1\right) \cos \theta_{b} \sin \left(\phi_{a}+\phi_{b}\right)\right\} \\
& \mathcal{A}_{220}^{010}=\int \mathrm{d} \Omega\left(\frac{1}{\Gamma} \frac{\mathrm{d} \Gamma}{\mathrm{d} \Omega}-\frac{1}{\bar{\Gamma}} \frac{\mathrm{d} \bar{\Gamma}}{\mathrm{d} \Omega}\right) \operatorname{sign}\left\{\cos \theta_{a} \sin \left(2 \phi_{a}+2 \phi_{b}\right)\right\}
\end{aligned}
$$

asymmetries (see tables 2 and 4). Note the latter two as well as $\mathcal{A}_{110}^{001}$ come proportional to the asymmetry parameter $\alpha_{a}$ which vanishes if the $a^{3 / 2} \rightarrow 1^{1 / 2} 2^{0}$ decay preserves parity, as $\Lambda(1520) \rightarrow p K$ does, being mediated by the strong interaction. A $\operatorname{sign}\left\{\left(1-9 \cos ^{2} \theta_{a}\right) \cos \theta_{b} \sin \left(\phi_{a}+\phi_{b}\right)\right\}$ asymmetry which is not independent of the $\operatorname{sign}\left\{\cos \theta_{b} \sin \left(\phi_{a}+\phi_{b}\right)\right\}$ and $\operatorname{sign}\left\{\left(3 \cos ^{2} \theta_{a}-1\right) \cos \theta_{b} \sin \left(\phi_{a}+\phi_{b}\right)\right\}$ ones has not been listed.

Let us also comment on the classical $\mathcal{A}_{110}^{000}$ asymmetry, based on $\operatorname{sign}\left\{\sin \left(\phi_{a}+\phi_{b}\right)\right\}$, which changes sign where the antisymmetry contraction of the four independent external particle momenta $\epsilon_{\mu \nu \rho \sigma} p_{1}^{\mu} p_{2}^{\nu} p_{3}^{\rho} p_{4}^{\sigma}$ does. Its use for studying CP violation in the decay of $\Lambda_{b}$ and $\Xi_{b}$ baryons was advocated in ref. [21]. We however stress that, contrarily to the four-body decay of spinless particles where it can play a significant role, it vanishes in the four four-body decays considered here, under the assumptions stated. Examining table 912 where these assumptions are relaxed, one realises such an asymmetry only appears proportional to the $\alpha_{b}$ asymmetry parameters in the $0^{1 / 2} \rightarrow a^{1 / 2,3 / 2} b^{1} \rightarrow 1^{1 / 2} 2^{0} 3^{1 / 2} 4^{1 / 2}$ decays. Even in such processes, its presence is therefore seen to require parity violation in 
the $b^{1} \rightarrow 3^{1 / 2} 4^{1 / 2}$ daughter decay (which would for instance be absent in electromagnetic $J / \psi \rightarrow \ell^{+} \ell^{-}$decays).

Following refs. [16-18], the LHCb collaboration measured the four

$$
\frac{1}{\Gamma} \int \mathrm{d} \Omega \frac{\mathrm{d} \Gamma}{\mathrm{d} \Omega} \operatorname{sign}\left\{\cos \Phi_{a, b}, \sin \Phi_{a, b}\right\}
$$

asymmetries in the $\Lambda_{b} \rightarrow \Lambda \varphi \rightarrow p \pi K^{+} K^{-}$decay [6]. The original definitions of those so-called special angles are easily seen to be equivalent to:

$$
\cos \Phi_{a}=\frac{\boldsymbol{n}_{\boldsymbol{a}} \cdot \boldsymbol{x}}{\sqrt{1-\left(\boldsymbol{n}_{\boldsymbol{a}} \cdot \boldsymbol{z}\right)^{2}}}, \quad \sin \Phi_{a}=\frac{\boldsymbol{n}_{\boldsymbol{a}} \cdot \boldsymbol{y}}{\sqrt{1-\left(\boldsymbol{n}_{\boldsymbol{a}} \cdot \boldsymbol{z}\right)^{2}}},
$$

and similarly for $a \leftrightarrow b$. Using $(\boldsymbol{x}, \boldsymbol{y}, \boldsymbol{z})^{T}=R(\phi, \theta, 0)\left(\boldsymbol{x}_{\boldsymbol{a}}, \boldsymbol{y}_{\boldsymbol{a}}, \boldsymbol{z}_{\boldsymbol{a}}\right)^{T}$,

$$
\left(\begin{array}{l}
\boldsymbol{x} \\
\boldsymbol{y} \\
\boldsymbol{z}
\end{array}\right)=\left(\begin{array}{ccc}
\cos \theta \cos \phi & -\sin \phi & \sin \theta \cos \phi \\
\cos \theta \sin \phi & \cos \phi & \sin \theta \sin \phi \\
-\sin \theta & 0 & \cos \theta
\end{array}\right)\left(\begin{array}{l}
\boldsymbol{x}_{\boldsymbol{a}} \\
\boldsymbol{y}_{\boldsymbol{a}} \\
\boldsymbol{z}_{\boldsymbol{a}}
\end{array}\right),
$$

as well as $(\boldsymbol{x}, \boldsymbol{y}, \boldsymbol{z})^{T}=R(\pi+\phi, \pi-\theta, 0)\left(\boldsymbol{x}_{\boldsymbol{b}}, \boldsymbol{y}_{\boldsymbol{b}}, \boldsymbol{z}_{\boldsymbol{b}}\right)^{T}$, one derives

$$
\begin{aligned}
& \cos \Phi_{a}=\frac{\cos \theta \cos \phi \sin \phi_{a}+\sin \phi \cos \phi_{a}}{\sqrt{1-\sin ^{2} \phi_{a} \sin ^{2} \theta}}, \quad \sin \Phi_{a}=\frac{\cos \theta \sin \phi \sin \phi_{a}-\cos \phi \cos \phi_{a}}{\sqrt{1-\sin ^{2} \phi_{a} \sin ^{2} \theta}}, \\
& \cos \Phi_{b}=\frac{\cos \theta \cos \phi \sin \phi_{b}-\sin \phi \cos \phi_{b}}{\sqrt{1-\sin ^{2} \phi_{b} \sin ^{2} \theta}}, \quad \sin \Phi_{b}=\frac{\cos \theta \sin \phi \sin \phi_{a}+\cos \phi \cos \phi_{b}}{\sqrt{1-\sin ^{2} \phi_{b} \sin ^{2} \theta}} .
\end{aligned}
$$

Such angular dependences do not appear in table 3. We therefore stress that these four asymmetries vanish identically in the $0^{1 / 2} \rightarrow a^{1 / 2} b^{1} \rightarrow 1^{1 / 2} 2^{0} 3^{1 / 2} 4^{1 / 2}$ process, when $\Lambda_{b}$ is produced by the strong interaction which preserves parity. Referring to table 1 , we note the same conclusion would also hold in $0^{1 / 2} \rightarrow a^{1 / 2} b^{1} \rightarrow 1^{1 / 2} 2^{0} 3^{1 / 2} 4^{1 / 2}$ processes like $\Lambda_{b} \rightarrow \Lambda J / \psi \rightarrow p \pi \mu^{+} \mu^{-}$. Relaxing the assumptions of our main text, tables 9 and 11 in appendix A inform us that, in both processes, asymmetries or moments based on $\cos \Phi_{a}$ and $\sin \Phi_{a}$ are respectively sensitive to the $\operatorname{Im}\left\{A_{+}^{*} A_{-}\right\} \alpha_{a} P_{x}$ and $\operatorname{Im}\left\{A_{+}^{*} A_{-}\right\} \alpha_{a} P_{y}$ combinations of production and decay amplitudes. In the $0^{1 / 2} \rightarrow a^{1 / 2} b^{1} \rightarrow 1^{1 / 2} 2^{0} 3^{1 / 2} 4^{1 / 2}$ decay, $\cos \Phi_{b}$ and $\sin \Phi_{b}$ asymmetries respectively provide access to $\left(\operatorname{Im}\left\{A_{+}^{*} B_{+}\right\}-\operatorname{Im}\left\{A_{-}^{*} B_{-}\right\}\right)$ $\alpha_{b} P_{x}$ and $\left(\operatorname{Im}\left\{A_{+}^{*} B_{+}\right\}-\operatorname{Im}\left\{A_{-}^{*} B_{-}\right\}\right) \alpha_{b} P_{y}$. They however vanish identically in the $0^{1 / 2} \rightarrow a^{1 / 2} b^{1} \rightarrow 1^{1 / 2} 2^{0} 3^{0} 4^{0}$ case.

\section{$4.2 \hat{\mathbf{T}}$-even angular asymmetries}

With a nonvanishing $\hat{\mathrm{T}}$-odd polarisation component $P_{z}$ produced by absorptive parts in the production amplitudes, one could also search for CP-odd phase differences between decay amplitudes that have identical strong phases through $\hat{\mathrm{T}}$-even angular asymmetries. In the four-body processes featuring an intermediate resonance $a$ of spin $1 / 2$, this is for 
instance possible with the

$$
\begin{aligned}
& \mathcal{A}_{010}^{001}=\int \mathrm{d} \Omega\left(\frac{1}{\Gamma} \frac{\mathrm{d} \Gamma}{\mathrm{d} \Omega}-\frac{1}{\bar{\Gamma}} \frac{\mathrm{d} \bar{\Gamma}}{\mathrm{d} \Omega}\right) \operatorname{sign}\left\{\cos \theta_{b} \sin \left(\phi_{b}\right)\right\}, \\
& \mathcal{A}_{110}^{101}=\int \mathrm{d} \Omega\left(\frac{1}{\Gamma} \frac{\mathrm{d} \Gamma}{\mathrm{d} \Omega}-\frac{1}{\bar{\Gamma}} \frac{\mathrm{d} \bar{\Gamma}}{\mathrm{d} \Omega}\right) \operatorname{sign}\left\{\cos \theta \cos \theta_{b} \sin \left(\phi_{a}+\phi_{b}\right)\right\}, \\
& \mathcal{A}_{010}^{011}=\int \mathrm{d} \Omega\left(\frac{1}{\Gamma} \frac{\mathrm{d} \Gamma}{\mathrm{d} \Omega}-\frac{1}{\bar{\Gamma}} \frac{\mathrm{d} \bar{\Gamma}}{\mathrm{d} \Omega}\right) \operatorname{sign}\left\{\cos \theta_{a} \cos \theta_{b} \sin \left(\phi_{b}\right)\right\}, \\
& \mathcal{A}_{100}^{000}=\int \mathrm{d} \Omega\left(\frac{1}{\Gamma} \frac{\mathrm{d} \Gamma}{\mathrm{d} \Omega}-\frac{1}{\bar{\Gamma}} \frac{\mathrm{d} \bar{\Gamma}}{\mathrm{d} \Omega}\right) \operatorname{sign}\left\{\sin \left(\phi_{a}\right)\right\}, \\
& \mathcal{A}_{120}^{000}=\int \mathrm{d} \Omega\left(\frac{1}{\Gamma} \frac{\mathrm{d} \Gamma}{\mathrm{d} \Omega}-\frac{1}{\bar{\Gamma}} \frac{\mathrm{d} \bar{\Gamma}}{\mathrm{d} \Omega}\right) \operatorname{sign}\left\{\sin \left(\phi_{a}+2 \phi_{b}\right)\right\},
\end{aligned}
$$

asymmetries (see tables 1 and 3). Only the first of these is not proportional to the asymmetry parameter $\alpha_{a}$, on top of $P_{z}$. Many more of such asymmetries can be constructed in the case $a$ is of spin $3 / 2$ and we refer the reader to the third blocks of tables 2 and 4 . The third blocks of tables 6 to 8 are relevant for three-body processes (table 5 has no such block). It is worth stressing here that the polarisation of the $\Lambda_{b}$ 's observed to decay to a $J / \psi \Lambda$ final state in the $\mathrm{LHCb}$ detector has been constrained to be smaller than $20 \%$ at the $2.7 \sigma$ level [5].

In principle, the above asymmetries could also be nonvanishing in the presence of $\mathrm{CP}$ violation in the production process, combined with strong phase differences between decay amplitudes. This is not expected to happen when the production process is dominated by the strong interaction but could also be checked experimentally by measuring various asymmetries. Since CP violation in production would cause $\left|P_{z}\right|$ to take slightly different values in the two conjugated processes, all the above asymmetries could potentially be nonvanishing. Moreover, the $\hat{\mathrm{T}}$-odd angular asymmetries giving access to terms proportional to $P_{z}$ (in the second blocks of our tables) would then be nonvanishing even in the absence of CP-even phase differences between decay amplitudes. In this sense, our tables would allow to interpret the patterns observed in the measurement of various asymmetries.

\section{Summary}

We have studied the angular distributions of some three- and four-body decays of spin- $1 / 2$ states, focusing on the discrete symmetry transformation properties of the different contributions. Some CP-odd asymmetries discussed in the literature have been shown to vanish identically in the decay chains considered. Special attention has been devoted to the two types of angular asymmetries that could serve to access small differences of CP-odd phases between decay amplitudes of identical CP-even phases. The first ones are $\hat{\mathrm{T}}$-odd angular asymmetries that are not proportional to a $\hat{\mathrm{T}}$-odd initial-state polarisation component $P_{z}$. The second ones are $\hat{\mathrm{T}}$-even angular asymmetries proportional to $P_{z}$. The latter do obviously not appear in the decay of spinless particles and are, on the other hand, the only way to access imaginary parts of decay amplitude interferences in the three-body decays of spinning particles (with unmeasured final-state spins). Conversely, it was stressed that some $\hat{\mathrm{T}}$-odd angular asymmetries only give access to the imaginary parts of production 
— and not decay — amplitude interferences. The $\hat{\mathrm{T}}$-odd angular asymmetries sensitive to imaginary parts of production amplitude interferences could serve to verify the assumption of $\mathrm{CP}$ conservation in production, without relying on nonvanishing differences of CP-even phases between either production or decay amplitudes. So eventually, comparing the measured patterns of asymmetries with the expectations provided here for specific resonant intermediate states could allow to decrypt the dynamical nature of the process scrutinized.

\section{Acknowledgments}

I am grateful to Yuval Grossman and Maurizio Martinelli for discussions on the topic treated here. Together with Christophe Grojean they also provided much valued comments on the manuscript of this paper.

\section{A Extended angular distributions}

We present below the distributions obtained by relaxing the hypotheses made in the main text. When parity is violated in the production of particle 0 , its $P_{x}$ and $P_{y}$ polarisation components can be nonvanishing. In three-body decays with a massive vector $b^{1}$ appearing in the final state, the $A_{ \pm}$amplitudes for which $\lambda_{b}=0$ can also be nonvanishing. Moreover, in the $b^{1} \rightarrow 3^{1 / 2} 4^{1 / 2}$ decay, parity violation and massive 3 , 4 fermions respectively produces terms proportional to:

$$
\begin{aligned}
\alpha_{b} & \equiv \frac{\left|M_{b}(+1 / 2,-1 / 2)\right|^{2}-\left|M_{b}(-1 / 2,+1 / 2)\right|^{2}}{\left|M_{b}(+1 / 2,-1 / 2)\right|^{2}+\left|M_{b}(-1 / 2,+1 / 2)\right|^{2}}, \quad \text { and } \\
\mu_{b} & \equiv \frac{\left|M_{b}(+1 / 2,+1 / 2)\right|^{2}+\left|M_{b}(-1 / 2,-1 / 2)\right|^{2}}{\left|M_{b}(+1 / 2,-1 / 2)\right|^{2}+\left|M_{b}(-1 / 2,+1 / 2)\right|^{2}} .
\end{aligned}
$$

Tables 9 to 16 respectively extend tables 1 to 8 with these additional contributions to the kinematic distributions. There, we used the $\left(\tilde{P}_{x}, \tilde{P}_{y}, \tilde{P}_{z}\right)^{T} \equiv R(\phi, \theta, 0)^{T}\left(P_{x}, P_{y}, P_{z}\right)^{T}$ polarisations along the $\boldsymbol{x}_{\boldsymbol{a}}, \boldsymbol{y}_{\boldsymbol{a}}$, and $\boldsymbol{z}_{\boldsymbol{a}}$ directions:

$$
\left(\begin{array}{c}
\tilde{P}_{x} \\
\tilde{P}_{y} \\
\tilde{P}_{z}
\end{array}\right)=\left(\begin{array}{ccc}
\cos \theta \cos \phi & \cos \theta \sin \phi & -\sin \theta \\
-\sin \phi & \cos \phi & 0 \\
\sin \theta \cos \phi & \sin \theta \sin \phi & \cos \theta
\end{array}\right)\left(\begin{array}{c}
P_{x} \\
P_{y} \\
P_{z}
\end{array}\right) .
$$

Referring to section 3 where the discrete symmetry properties of the different quantities above were derived, one sees that $\tilde{P}_{x}$ is P-even- $\hat{\mathrm{T}}$-odd while $\tilde{P}_{y}$ and $\tilde{P}_{z}$ are both P-odd- $\hat{\mathrm{T}}$-even. 


\begin{tabular}{|c|c|c|}
\hline$+3 / 2$ & $\left(\left|A_{+}\right|^{2}-\left|A_{-}\right|^{2}\right)+\left(\left|A_{+}\right|^{2}+\left|A_{-}\right|^{2}\right) \tilde{P}_{z}$ & $\left(\sin ^{2} \theta_{b}+2 \cos ^{2} \theta_{b} \mu_{b}\right) \cos \theta_{a} \alpha_{a}$ \\
\hline$+3 / 2$ & $\left(\left|A_{+}\right|^{2}-\left|A_{-}\right|^{2}\right) \tilde{P}_{z}+\left(\left|A_{+}\right|^{2}+\left|A_{-}\right|^{2}\right)$ & $\left(\sin ^{2} \theta_{b}+2 \cos ^{2} \theta_{b} \mu_{b}\right)$ \\
\hline$-3 / 4$ & $\left(\left|B_{+}\right|^{2}-\left|B_{-}\right|^{2}\right) \tilde{P}_{z}-\left(\left|B_{+}\right|^{2}+\left|B_{-}\right|^{2}\right)$ & $2 \cos \theta_{a} \cos \theta_{b} \alpha_{a} \alpha_{b}+\left(1+2 \sin ^{2} \theta_{b} \mu_{b}+\cos ^{2} \theta_{b}\right)$ \\
\hline$+3 / 4$ & $\left(\left|B_{+}\right|^{2}-\left|B_{-}\right|^{2}\right)-\left(\left|B_{+}\right|^{2}+\left|B_{-}\right|^{2}\right) \tilde{P}_{z}$ & $2 \cos \theta_{b} \alpha_{b}+\left(1+2 \sin ^{2} \theta_{b} \mu_{b}+\cos ^{2} \theta_{b}\right) \cos \theta_{a} \alpha_{a}$ \\
\hline+3 & $\operatorname{Re}\left\{A_{+}^{*} A_{-}\right\} \tilde{P}_{x}-\operatorname{Im}\left\{A_{+}^{*} A_{-}\right\} \tilde{P}_{y}$ & $\left(\sin ^{2} \theta_{b}+2 \cos ^{2} \theta_{b} \mu_{b}\right) \sin \theta_{a} \alpha_{a}$ \\
\hline$+3 / 2 \sqrt{2}$ & $\left(\operatorname{Re}\left\{A_{+}^{*} B_{+}\right\}-\operatorname{Re}\left\{A_{-}^{*} B_{-}\right\}\right) \tilde{P}_{x}-\left(\operatorname{Im}\left\{A_{+}^{*} B_{+}\right\}+\operatorname{Im}\left\{A_{-}^{*} B_{-}\right\}\right) \tilde{P}_{y}$ & $2 \cos \theta_{a} \sin \theta_{b} \alpha_{a} \alpha_{b}+\left(1-2 \mu_{b}\right) \sin 2 \theta_{b}$ \\
\hline$+3 / 2 \sqrt{2}$ & $\left(\operatorname{Re}\left\{A_{+}^{*} B_{+}\right\}+\operatorname{Re}\left\{A_{-}^{*} B_{-}\right\}\right) \tilde{P}_{x}-\left(\operatorname{Im}\left\{A_{+}^{*} B_{+}\right\}-\operatorname{Im}\left\{A_{-}^{*} B_{-}\right\}\right) \tilde{P}_{y}$ & $2 \sin \theta_{b} \alpha_{b}+\left(1-2 \mu_{b}\right) \cos \theta_{a} \sin 2 \theta_{b} \alpha_{a}$ \\
\hline$+3 / 2$ & $\operatorname{Re}\left\{B_{+}^{*} B_{-}\right\} \tilde{P}_{x}+\operatorname{Im}\left\{B_{+}^{*} B_{-}\right\} \tilde{P}_{y}$ & $\left(1-2 \mu_{b}\right) \sin \theta_{a} \sin ^{2} \theta_{b} \alpha_{a}$ \\
\hline$-3 / 2 \sqrt{2}$ & $\left(\operatorname{Re}\left\{A_{+}^{*} B_{-}\right\}-\operatorname{Re}\left\{A_{-}^{*} B_{+}\right\}\right)+\left(\operatorname{Re}\left\{A_{+}^{*} B_{-}\right\}+\operatorname{Re}\left\{A_{-}^{*} B_{+}\right\}\right) \tilde{P}_{z} \quad \cos \left(\phi_{a}+\phi_{b}\right)$ & $\left(1-2 \mu_{b}\right) \sin \theta_{a} \sin 2 \theta_{b} \alpha_{a}$ \\
\hline$+3 / \sqrt{2}$ & $\left(\operatorname{Re}\left\{A_{+}^{*} B_{-}\right\}-\operatorname{Re}\left\{A_{-}^{*} B_{+}\right\}\right) \tilde{P}_{z}+\left(\operatorname{Re}\left\{A_{+}^{*} B_{-}\right\}+\operatorname{Re}\left\{A_{-}^{*} B_{+}\right\}\right) \quad \cos \left(\phi_{a}+\phi_{b}\right)$ & $\sin \theta_{a} \sin \theta_{b} \alpha_{a} \alpha_{b}$ \\
\hline+3 & $\operatorname{Re}\left\{A_{+}^{*} A_{-}\right\} \tilde{P}_{y}+\operatorname{Im}\left\{A_{+}^{*} A_{-}\right\} \tilde{P}_{x}$ & $\left(\sin ^{2} \theta_{b}+2 \cos ^{2} \theta_{b} \mu_{b}\right) \sin \theta_{a} \alpha_{a}$ \\
\hline$-3 / 2 \sqrt{2}$ & $\left(\operatorname{Re}\left\{A_{+}^{*} B_{+}\right\}-\operatorname{Re}\left\{A_{-}^{*} B_{-}\right\}\right) \tilde{P}_{y}+\left(\operatorname{Im}\left\{A_{+}^{*} B_{+}\right\}+\operatorname{Im}\left\{A_{-}^{*} B_{-}\right\}\right) \tilde{P}_{x}$ & $2 \cos \theta_{a} \sin \theta_{b} \alpha_{a} \alpha_{b}+\left(1-2 \mu_{b}\right) \sin 2 \theta_{b}$ \\
\hline$-3 / 2 \sqrt{2}$ & $\left(\operatorname{Re}\left\{A_{+}^{*} B_{+}\right\}+\operatorname{Re}\left\{A_{-}^{*} B_{-}\right\}\right) \tilde{P}_{y}+\left(\operatorname{Im}\left\{A_{+}^{*} B_{+}\right\}-\operatorname{Im}\left\{A_{-}^{*} B_{-}\right\}\right) \tilde{P}_{x}$ & $2 \sin \theta_{b} \alpha_{b}+\left(1-2 \mu_{b}\right) \cos \theta_{a} \sin 2 \theta_{b} \alpha_{a}$ \\
\hline$-3 / 2$ & $\operatorname{Re}\left\{B_{+}^{*} B_{-}\right\} \tilde{P}_{y}-\operatorname{Im}\left\{B_{+}^{*} B_{-}\right\} \tilde{P}_{x}$ & $\left(1-2 \mu_{b}\right) \sin \theta_{a} \sin ^{2} \theta_{b} \alpha_{a}$ \\
\hline$-3 / 2 \sqrt{2}$ & $\left(\operatorname{Im}\left\{A_{+}^{*} B_{-}\right\}-\operatorname{Im}\left\{A_{-}^{*} B_{+}\right\}\right) \tilde{P}_{z}+\left(\operatorname{Im}\left\{A_{+}^{*} B_{-}\right\}+\operatorname{Im}\left\{A_{-}^{*} B_{+}\right\}\right) \quad \sin \left(\phi_{a}+\phi_{b}\right)$ & $\left(1-2 \mu_{b}\right) \sin \theta_{a} \sin 2 \theta_{b} \alpha_{a}$ \\
\hline$+3 / \sqrt{2}$ & $\left(\operatorname{Im}\left\{A_{+}^{*} B_{-}\right\}-\operatorname{Im}\left\{A_{-}^{*} B_{+}\right\}\right)+\left(\operatorname{Im}\left\{A_{+}^{*} B_{-}\right\}+\operatorname{Im}\left\{A_{-}^{*} B_{+}\right\}\right) \tilde{P}_{z}$ & $\sin \theta_{a} \sin \theta_{b} \alpha_{a} \alpha_{b}$ \\
\hline
\end{tabular}

Table 9. All contributions to $0^{1 / 2} \rightarrow a^{1 / 2} b^{1} \rightarrow 1^{1 / 2} 2^{0} 3^{1 / 2} 4^{1 / 2}$ angular distribution which appear when the assumptions leading to table 1 are relaxed, so that $P_{x}, P_{y}, \alpha_{b}$ or $\mu_{b}$ defined in the text are nonvanishing. We have defined $\left(\tilde{P}_{x}, \tilde{P}_{y}, \tilde{P}_{z}\right) \equiv R(\phi, \theta, 0)^{T}\left(P_{x}, P_{y}, P_{z}\right)$. 


\begin{tabular}{|c|c|c|c|}
\hline$-3 / 4$ & \multirow{2}{*}{\multicolumn{2}{|c|}{$\begin{array}{l}\left(\left|A_{+}\right|^{2}-\left|A_{-}\right|^{2}\right)+\left(\left|A_{+}\right|^{2}+\left|A_{-}\right|^{2}\right) \tilde{P}_{z} \\
\left(\left|A_{+}\right|^{2}-\left|A_{-}\right|^{2}\right) \tilde{P}_{z}+\left(\left|A_{+}\right|^{2}+\left|A_{-}\right|^{2}\right)\end{array}$}} & $\left(\sin ^{2} \theta_{b}+2 \cos ^{2} \theta_{b} \mu_{b}\right)\left(5-9 \cos ^{2} \theta_{a}\right) \cos \theta_{a} \alpha_{a}$ \\
\hline$+3 / 4$ & & & $\left(\sin ^{2} \theta_{b}+2 \cos ^{2} \theta_{b} \mu_{b}\right)\left(1+3 \cos ^{2} \theta_{a}\right)$ \\
\hline$+9 / 8$ & \multicolumn{2}{|l|}{$\left(3\left|B_{+}\right|^{2}-3\left|B_{-}\right|^{2}-\left|C_{+}\right|^{2}+\left|C_{-}\right|^{2}\right)-\left(3\left|B_{+}\right|^{2}+3\left|B_{-}\right|^{2}+\left|C_{+}\right|^{2}+\left|C_{-}\right|^{2}\right) \tilde{P}_{z}$} & $\left(1+2 \sin ^{2} \theta_{b} \mu_{b}+\cos ^{2} \theta_{b}\right) \cos ^{3} \theta_{a} \alpha_{a}$ \\
\hline$-9 / 8$ & \multicolumn{2}{|l|}{$\left(\left|B_{+}\right|^{2}-\left|B_{-}\right|^{2}+\left|C_{+}\right|^{2}-\left|C_{-}\right|^{2}\right) \tilde{P}_{z}-\left(\left|B_{+}\right|^{2}+\left|B_{-}\right|^{2}-\left|C_{+}\right|^{2}-\left|C_{-}\right|^{2}\right)$} & $\left(1+2 \sin ^{2} \theta_{b} \mu_{b}+\cos ^{2} \theta_{b}\right) \cos ^{2} \theta_{a}$ \\
\hline$-3 / 8 \quad($ & \multicolumn{2}{|l|}{$\left(5\left|B_{+}\right|^{2}-5\left|B_{-}\right|^{2}-3\left|C_{+}\right|^{2}+3\left|C_{-}\right|^{2}\right)-\left(5\left|B_{+}\right|^{2}+5\left|B_{-}\right|^{2}+3\left|C_{+}\right|^{2}+3\left|C_{-}\right|^{2}\right) \tilde{P}_{z}$} & $\left(1+2 \sin ^{2} \theta_{b} \mu_{b}+\cos ^{2} \theta_{b}\right) \cos \theta_{a} \alpha_{a}$ \\
\hline$-3 / 8$ & \multicolumn{2}{|l|}{$\left(\left|B_{+}\right|^{2}-\left|B_{-}\right|^{2}-3\left|C_{+}\right|^{2}+3\left|C_{-}\right|^{2}\right) \tilde{P}_{z}-\left(\left|B_{+}\right|^{2}+\left|B_{-}\right|^{2}+3\left|C_{+}\right|^{2}+3\left|C_{-}\right|^{2}\right)$} & $\left(1+2 \sin ^{2} \theta_{b} \mu_{b}+\cos ^{2} \theta_{b}\right)$ \\
\hline$-9 / 4$ & \multicolumn{2}{|l|}{$\left(3\left|B_{+}\right|^{2}-3\left|B_{-}\right|^{2}+\left|C_{+}\right|^{2}-\left|C_{-}\right|^{2}\right) \tilde{P}_{z}-\left(3\left|B_{+}\right|^{2}+3\left|B_{-}\right|^{2}-\left|C_{+}\right|^{2}-\left|C_{-}\right|^{2}\right)$} & $\cos ^{3} \theta_{a} \cos \theta_{b} \alpha_{a} \alpha_{b}$ \\
\hline$+9 / 4$ & \multicolumn{2}{|l|}{$\left(\left|B_{+}\right|^{2}-\left|B_{-}\right|^{2}-\left|C_{+}\right|^{2}+\left|C_{-}\right|^{2}\right)-\left(\left|B_{+}\right|^{2}+\left|B_{-}\right|^{2}+\left|C_{+}\right|^{2}+\left|C_{-}\right|^{2}\right) \tilde{P}_{z}$} & $\cos ^{2} \theta_{a} \cos \theta_{b} \alpha_{b}$ \\
\hline$+3 / 4 \quad($ & \multicolumn{2}{|l|}{$\left(5\left|B_{+}\right|^{2}-5\left|B_{-}\right|^{2}+3\left|C_{+}\right|^{2}-3\left|C_{-}\right|^{2}\right) \tilde{P}_{z}-\left(5\left|B_{+}\right|^{2}+5\left|B_{-}\right|^{2}-3\left|C_{+}\right|^{2}-3\left|C_{-}\right|^{2}\right)$} & $\cos \theta_{a} \cos \theta_{b} \alpha_{a} \alpha_{b}$ \\
\hline$+3 / 4$ & \multicolumn{2}{|l|}{$\left(\left|B_{+}\right|^{2}-\left|B_{-}\right|^{2}+3\left|C_{+}\right|^{2}-3\left|C_{-}\right|^{2}\right)-\left(\left|B_{+}\right|^{2}+\left|B_{-}\right|^{2}-3\left|C_{+}\right|^{2}-3\left|C_{-}\right|^{2}\right) \tilde{P}_{z}$} & $\cos \theta_{b} \alpha_{b}$ \\
\hline$-3 / 2$ & $\operatorname{Re}\left\{A_{+}^{*} A_{-}\right\} \tilde{P}_{x}-\operatorname{Im}\left\{A_{+}^{*} A_{-}\right\} \tilde{P}_{y}$ & $\cos \phi_{a}$ & $\left(\sin ^{2} \theta_{b}+2 \cos ^{2} \theta_{b} \mu_{b}\right)\left(1-9 \cos ^{2} \theta_{a}\right) \sin \theta_{a} \alpha_{a}$ \\
\hline$-3 \sqrt{3} / 4$ & $\left(\operatorname{Re}\left\{B_{+}^{*} C_{+}\right\}-\operatorname{Re}\left\{B_{-}^{*} C_{-}\right\}\right) \tilde{P}_{x}+\left(\operatorname{Im}\left\{B_{+}^{*} C_{+}\right\}+\operatorname{Im}\left\{B_{-}^{*} C_{-}\right\}\right) \tilde{P}_{y}$ & $\cos \phi_{a}$ & $\left(1+2 \sin ^{2} \theta_{b} \mu_{b}+\cos ^{2} \theta_{b}\right) \sin 2 \theta_{a}-2\left(1-3 \cos ^{2} \theta_{a}\right) \sin \theta_{a} \cos \theta_{b} \alpha_{a} \alpha_{b}$ \\
\hline$-3 \sqrt{3} / 4$ & $\left(\operatorname{Re}\left\{B_{+}^{*} C_{+}\right\}+\operatorname{Re}\left\{B_{-}^{*} C_{-}\right\}\right) \tilde{P}_{x}+\left(\operatorname{Im}\left\{B_{+}^{*} C_{+}\right\}-\operatorname{Im}\left\{B_{-}^{*} C_{-}\right\}\right) \tilde{P}_{y}$ & $\cos \phi_{a}$ & $2 \sin 2 \theta_{a} \cos \theta_{b} \alpha_{b}-\left(1+2 \sin ^{2} \theta_{b} \mu_{b}+\cos ^{2} \theta_{b}\right)\left(1-3 \cos ^{2} \theta_{a}\right) \sin \theta_{a} \alpha_{a}$ \\
\hline$-3 / 4 \sqrt{2}$ & $\left(\operatorname{Re}\left\{A_{+}^{*} B_{+}\right\}+\operatorname{Re}\left\{A_{-}^{*} B_{-}\right\}\right) \tilde{P}_{x}-\left(\operatorname{Im}\left\{A_{+}^{*} B_{+}\right\}-\operatorname{Im}\left\{A_{-}^{*} B_{-}\right\}\right) \tilde{P}_{y}$ & $\cos \phi_{b}$ & $\left(1-2 \mu_{b}\right)\left(5-9 \cos ^{2} \theta_{a}\right) \cos \theta_{a} \sin 2 \theta_{b} \alpha_{a}-2\left(1+3 \cos ^{2} \theta_{a}\right) \sin \theta_{b} \alpha_{b}$ \\
\hline$+3 / 4 \sqrt{2}$ & $\left(\operatorname{Re}\left\{A_{+}^{*} B_{+}\right\}-\operatorname{Re}\left\{A_{-}^{*} B_{-}\right\}\right) \tilde{P}_{x}-\left(\operatorname{Im}\left\{A_{+}^{*} B_{+}\right\}+\operatorname{Im}\left\{A_{-}^{*} B_{-}\right\}\right) \tilde{P}_{y}$ & $\cos \phi_{b}$ & $\left(1-2 \mu_{b}\right)\left(1+3 \cos ^{2} \theta_{a}\right) \sin 2 \theta_{b}-2\left(5-9 \cos ^{2} \theta_{a}\right) \cos \theta_{a} \sin \theta_{b} \alpha_{a} \alpha_{b}$ \\
\hline$-9 / 4$ & $\operatorname{Re}\left\{C_{+}^{*} C_{-}\right\} \tilde{P}_{x}-\operatorname{Im}\left\{C_{+}^{*} C_{-}\right\} \tilde{P}_{y}$ & $\cos \left(3 \phi_{a}+2 \phi_{b}\right)$ & $\left(1-2 \mu_{b}\right) \sin \theta_{a}^{3} \sin ^{2} \theta_{b} \alpha_{a}$ \\
\hline$+9 \sqrt{3} / 4$ & $\left(\operatorname{Re}\left\{B_{+}^{*} C_{-}\right\}-\operatorname{Re}\left\{B_{-}^{*} C_{+}\right\}\right)-\left(\operatorname{Re}\left\{B_{+}^{*} C_{-}\right\}+\operatorname{Re}\left\{B_{-}^{*} C_{+}\right\}\right) \tilde{P}_{z}$ & $\cos \left(2 \phi_{a}+2 \phi_{b}\right)$ & $\left(1-2 \mu_{b}\right) \sin \theta_{a}^{2} \cos \theta_{a} \sin ^{2} \theta_{b} \alpha_{a}$ \\
\hline$+3 \sqrt{3} / 4$ & $\left(\operatorname{Re}\left\{B_{+}^{*} C_{-}\right\}-\operatorname{Re}\left\{B_{-}^{*} C_{+}\right\}\right) \tilde{P}_{z}-\left(\operatorname{Re}\left\{B_{+}^{*} C_{-}\right\}+\operatorname{Re}\left\{B_{-}^{*} C_{+}\right\}\right)$ & $\cos \left(2 \phi_{a}+2 \phi_{b}\right)$ & $\left(1-2 \mu_{b}\right) \sin \theta_{a}^{2} \sin ^{2} \theta_{b}$ \\
\hline$+3 \sqrt{2 / 3} / 4$ & $\left(\operatorname{Re}\left\{A_{+}^{*} C_{-}\right\}-\operatorname{Re}\left\{A_{-}^{*} C_{+}\right\}\right) \tilde{P}_{x}-\left(\operatorname{Im}\left\{A_{+}^{*} C_{-}\right\}+\operatorname{Im}\left\{A_{-}^{*} C_{+}\right\}\right) \tilde{P}_{y}$ & $\cos \left(2 \phi_{a}+\phi_{b}\right)$ & $6 \sin \theta_{a}^{2} \cos \theta_{a} \sin \theta_{b} \alpha_{a} \alpha_{b}+\left(1-2 \mu_{b}\right) \sin \theta_{a}^{2} \sin 2 \theta_{b}$ \\
\hline$-3 \sqrt{2 / 3} / 4$ & $\left(\operatorname{Re}\left\{A_{+}^{*} C_{-}\right\}+\operatorname{Re}\left\{A_{-}^{*} C_{+}\right\}\right) \tilde{P}_{x}-\left(\operatorname{Im}\left\{A_{+}^{*} C_{-}\right\}-\operatorname{Im}\left\{A_{-}^{*} C_{+}\right\}\right) \tilde{P}_{y}$ & $\cos \left(2 \phi_{a}+\phi_{b}\right)$ & $2 \sin \theta_{a}^{2} \sin \theta_{b} \alpha_{b}+3\left(1-2 \mu_{b}\right) \sin \theta_{a}^{2} \cos \theta_{a} \sin 2 \theta_{b} \alpha_{a}$ \\
\hline$-3 / 4$ & $\operatorname{Re}\left\{B_{+}^{*} B_{-}\right\} \tilde{P}_{x}+\operatorname{Im}\left\{B_{+}^{*} B_{-}\right\} \tilde{P}_{y}$ & $\cos \left(\phi_{a}+2 \phi_{b}\right)$ & $\left(1-2 \mu_{b}\right)\left(1-9 \cos ^{2} \theta_{a}\right) \sin \theta_{a} \sin ^{2} \theta_{b} \alpha_{a}$ \\
\hline$-3 / 2 \sqrt{2}$ & $\left(\operatorname{Re}\left\{A_{+}^{*} B_{-}\right\}-\operatorname{Re}\left\{A_{-}^{*} B_{+}\right\}\right) \tilde{P}_{z}+\left(\operatorname{Re}\left\{A_{+}^{*} B_{-}\right\}+\operatorname{Re}\left\{A_{-}^{*} B_{+}\right\}\right)$ & $\cos \left(\phi_{a}+\phi_{b}\right)$ & $\left(1-9 \cos ^{2} \theta_{a}\right) \sin \theta_{a} \sin \theta_{b} \alpha_{a} \alpha_{b}$ \\
\hline$+3 / 4 \sqrt{2}$ & $\left(\operatorname{Re}\left\{A_{+}^{*} B_{-}\right\}-\operatorname{Re}\left\{A_{-}^{*} B_{+}\right\}\right)+\left(\operatorname{Re}\left\{A_{+}^{*} B_{-}\right\}+\operatorname{Re}\left\{A_{-}^{*} B_{+}\right\}\right) \tilde{P}_{z}$ & $\cos \left(\phi_{a}+\phi_{b}\right)$ & $\left(1-2 \mu_{b}\right)\left(1-9 \cos ^{2} \theta_{a}\right) \sin \theta_{a} \sin 2 \theta_{b} \alpha_{a}$ \\
\hline$-3 \sqrt{2 / 3} / 4$ & $\left(\operatorname{Re}\left\{A_{+}^{*} C_{+}\right\}-\operatorname{Re}\left\{A_{-}^{*} C_{-}\right\}\right) \tilde{P}_{z}+\left(\operatorname{Re}\left\{A_{+}^{*} C_{+}\right\}+\operatorname{Re}\left\{A_{-}^{*} C_{-}\right\}\right)$ & $\cos \left(\phi_{a}+\phi_{b}\right)$ & $\left(1-2 \mu_{b}\right) \sin 2 \theta_{a} \sin 2 \theta_{b}-2\left(1-3 \cos ^{2} \theta_{a}\right) \sin \theta_{a} \sin \theta_{b} \alpha_{a} \alpha_{b}$ \\
\hline$-3 \sqrt{2 / 3} / 4$ & $\left(\operatorname{Re}\left\{A_{+}^{*} C_{+}\right\}-\operatorname{Re}\left\{A_{-}^{*} C_{-}\right\}\right)+\left(\operatorname{Re}\left\{A_{+}^{*} C_{+}\right\}+\operatorname{Re}\left\{A_{-}^{*} C_{-}\right\}\right) \tilde{P}_{z}$ & $\cos \left(\phi_{a}+\phi_{b}\right)$ & $2 \sin 2 \theta_{a} \sin \theta_{b} \alpha_{b}-\left(1-2 \mu_{b}\right)\left(1-3 \cos ^{2} \theta_{a}\right) \sin \theta_{a} \sin 2 \theta_{b} \alpha_{a}$ \\
\hline$-3 / 2$ & $\operatorname{Re}\left\{A_{+}^{*} A_{-}\right\} \tilde{P}_{y}+\operatorname{Im}\left\{A_{+}^{*} A_{-}\right\} \tilde{P}_{x}$ & $\sin \phi_{a}$ & $\left(\sin ^{2} \theta_{b}+2 \cos ^{2} \theta_{b} \mu_{b}\right)\left(1-9 \cos ^{2} \theta_{a}\right) \sin \theta_{a} \alpha_{a}$ \\
\hline$-3 \sqrt{3} / 4$ & $\left(\operatorname{Re}\left\{B_{+}^{*} C_{+}\right\}-\operatorname{Re}\left\{B_{-}^{*} C_{-}\right\}\right) \tilde{P}_{y}-\left(\operatorname{Im}\left\{B_{+}^{*} C_{+}\right\}+\operatorname{Im}\left\{B_{-}^{*} C_{-}\right\}\right) \tilde{P}_{x}$ & $\sin \phi_{a}$ & $\left(1+2 \sin ^{2} \theta_{b} \mu_{b}+\cos ^{2} \theta_{b}\right) \sin 2 \theta_{a}-2\left(1-3 \cos ^{2} \theta_{a}\right) \sin \theta_{a} \cos \theta_{b} \alpha_{a} \alpha_{b}$ \\
\hline$-3 \sqrt{3} / 4$ & $\left(\operatorname{Re}\left\{B_{+}^{*} C_{+}\right\}+\operatorname{Re}\left\{B_{-}^{*} C_{-}\right\}\right) \tilde{P}_{y}-\left(\operatorname{Im}\left\{B_{+}^{*} C_{+}\right\}-\operatorname{Im}\left\{B_{-}^{*} C_{-}\right\}\right) \tilde{P}_{x}$ & $\sin \phi_{a}$ & $2 \sin 2 \theta_{a} \cos \theta_{b} \alpha_{b}-\left(1+2 \sin ^{2} \theta_{b} \mu_{b}+\cos ^{2} \theta_{b}\right)\left(1-3 \cos ^{2} \theta_{a}\right) \sin \theta_{a} \alpha_{a}$ \\
\hline$+3 / 4 \sqrt{2}$ & $\left(\operatorname{Re}\left\{A_{+}^{*} B_{+}\right\}+\operatorname{Re}\left\{A_{-}^{*} B_{-}\right\}\right) \tilde{P}_{y}+\left(\operatorname{Im}\left\{A_{+}^{*} B_{+}\right\}-\operatorname{Im}\left\{A_{-}^{*} B_{-}\right\}\right) \tilde{P}_{x}$ & $\sin \phi_{b}$ & $\left(1-2 \mu_{b}\right)\left(5-9 \cos ^{2} \theta_{a}\right) \cos \theta_{a} \sin 2 \theta_{b} \alpha_{a}-2\left(1+3 \cos ^{2} \theta_{a}\right) \sin \theta_{b} \alpha_{b}$ \\
\hline$-3 / 4 \sqrt{2}$ & $\left(\operatorname{Re}\left\{A_{+}^{*} B_{+}\right\}-\operatorname{Re}\left\{A_{-}^{*} B_{-}\right\}\right) \tilde{P}_{y}+\left(\operatorname{Im}\left\{A_{+}^{*} B_{+}\right\}+\operatorname{Im}\left\{A_{-}^{*} B_{-}\right\}\right) \tilde{P}_{x}$ & $\sin \phi_{b}$ & $\left(1-2 \mu_{b}\right)\left(1+3 \cos ^{2} \theta_{a}\right) \sin 2 \theta_{b}-2\left(5-9 \cos ^{2} \theta_{a}\right) \cos \theta_{a} \sin \theta_{b} \alpha_{a} \alpha_{b}$ \\
\hline$-9 / 4$ & $\operatorname{Re}\left\{C_{+}^{*} C_{-}\right\} \tilde{P}_{y}+\operatorname{Im}\left\{C_{+}^{*} C_{-}\right\} \tilde{P}_{x}$ & $\sin \left(3 \phi_{a}+2 \phi_{b}\right)$ & $\left(1-2 \mu_{b}\right) \sin \theta_{a}^{3} \sin ^{2} \theta_{b} \alpha_{a}$ \\
\hline$-9 \sqrt{3} / 4$ & $\left(\operatorname{Im}\left\{B_{+}^{*} C_{-}\right\}-\operatorname{Im}\left\{B_{-}^{*} C_{+}\right\}\right) \tilde{P}_{z}-\left(\operatorname{Im}\left\{B_{+}^{*} C_{-}\right\}+\operatorname{Im}\left\{B_{-}^{*} C_{+}\right\}\right)$ & $\sin \left(2 \phi_{a}+2 \phi_{b}\right)$ & $\left(1-2 \mu_{b}\right) \sin \theta_{a}^{2} \cos \theta_{a} \sin ^{2} \theta_{b} \alpha_{a}$ \\
\hline$-3 \sqrt{3} / 4$ & $\left(\operatorname{Im}\left\{B_{+}^{*} C_{-}\right\}-\operatorname{Im}\left\{B_{-}^{*} C_{+}\right\}\right)-\left(\operatorname{Im}\left\{B_{+}^{*} C_{-}\right\}+\operatorname{Im}\left\{B_{-}^{*} C_{+}\right\}\right) \tilde{P}_{z}$ & $\sin \left(2 \phi_{a}+2 \phi_{b}\right)$ & $\left(1-2 \mu_{b}\right) \sin \theta_{a}^{2} \sin ^{2} \theta_{b}$ \\
\hline$+3 \sqrt{2 / 3} / 4$ & $\left(\operatorname{Re}\left\{A_{+}^{*} C_{-}\right\}-\operatorname{Re}\left\{A_{-}^{*} C_{+}\right\}\right) \tilde{P}_{y}+\left(\operatorname{Im}\left\{A_{+}^{*} C_{-}\right\}+\operatorname{Im}\left\{A_{-}^{*} C_{+}\right\}\right) \tilde{P}_{x}$ & $\sin \left(2 \phi_{a}+\phi_{b}\right)$ & $6 \sin \theta_{a}^{2} \cos \theta_{a} \sin \theta_{b} \alpha_{a} \alpha_{b}+\left(1-2 \mu_{b}\right) \sin \theta_{a}^{2} \sin 2 \theta_{b}$ \\
\hline$-3 \sqrt{2 / 3} / 4$ & $\left(\operatorname{Re}\left\{A_{+}^{*} C_{-}\right\}+\operatorname{Re}\left\{A_{-}^{*} C_{+}\right\}\right) \tilde{P}_{y}+\left(\operatorname{Im}\left\{A_{+}^{*} C_{-}\right\}-\operatorname{Im}\left\{A_{-}^{*} C_{+}\right\}\right) \tilde{P}_{x}$ & $\sin \left(2 \phi_{a}+\phi_{b}\right)$ & $2 \sin \theta_{a}^{2} \sin \theta_{b} \alpha_{b}+3\left(1-2 \mu_{b}\right) \sin \theta_{a}^{2} \cos \theta_{a} \sin 2 \theta_{b} \alpha_{a}$ \\
\hline$+3 / 4$ & $\operatorname{Re}\left\{B_{+}^{*} B_{-}\right\} \tilde{P}_{y}-\operatorname{Im}\left\{B_{+}^{*} B_{-}\right\} \tilde{P}_{x}$ & $\sin \left(\phi_{a}+2 \phi_{b}\right)$ & $\left(1-2 \mu_{b}\right)\left(1-9 \cos ^{2} \theta_{a}\right) \sin \theta_{a} \sin ^{2} \theta_{b} \alpha_{a}$ \\
\hline$-3 / 2 \sqrt{2}$ & $\left(\operatorname{Im}\left\{A_{+}^{*} B_{-}\right\}-\operatorname{Im}\left\{A_{-}^{*} B_{+}\right\}\right)+\left(\operatorname{Im}\left\{A_{+}^{*} B_{-}\right\}+\operatorname{Im}\left\{A_{-}^{*} B_{+}\right\}\right) \tilde{P}_{z}$ & $\sin \left(\phi_{a}+\phi_{b}\right)$ & $\left(1-9 \cos ^{2} \theta_{a}\right) \sin \theta_{a} \sin \theta_{b} \alpha_{a} \alpha_{b}$ \\
\hline$+3 / 4 \sqrt{2}$ & $\left(\operatorname{Im}\left\{A_{+}^{*} B_{-}\right\}-\operatorname{Im}\left\{A_{-}^{*} B_{+}\right\}\right) \tilde{P}_{z}+\left(\operatorname{Im}\left\{A_{+}^{*} B_{-}\right\}+\operatorname{Im}\left\{A_{-}^{*} B_{+}\right\}\right)$ & $\sin \left(\phi_{a}+\phi_{b}\right)$ & $\left(1-2 \mu_{b}\right)\left(1-9 \cos ^{2} \theta_{a}\right) \sin \theta_{a} \sin 2 \theta_{b} \alpha_{a}$ \\
\hline$+3 \sqrt{2 / 3} / 4$ & $\left(\operatorname{Im}\left\{A_{+}^{*} C_{+}\right\}-\operatorname{Im}\left\{A_{-}^{*} C_{-}\right\}\right)+\left(\operatorname{Im}\left\{A_{+}^{*} C_{+}\right\}+\operatorname{Im}\left\{A_{-}^{*} C_{-}\right\}\right) \tilde{P}_{z}$ & $\sin \left(\phi_{a}+\phi_{b}\right)$ & $\left(1-2 \mu_{b}\right) \sin 2 \theta_{a} \sin 2 \theta_{b}-2\left(1-3 \cos ^{2} \theta_{a}\right) \sin \theta_{a} \sin \theta_{b} \alpha_{a} \alpha_{b}$ \\
\hline$+3 \sqrt{2 / 3} / 4$ & $\left(\operatorname{Im}\left\{A_{+}^{*} C_{+}\right\}-\operatorname{Im}\left\{A_{-}^{*} C_{-}\right\}\right) \tilde{P}_{z}+\left(\operatorname{Im}\left\{A_{+}^{*} C_{+}\right\}+\operatorname{Im}\left\{A_{-}^{*} C_{-}\right\}\right)$ & $\sin \left(\phi_{a}+\phi_{b}\right)$ & $2 \sin 2 \theta_{a} \sin \theta_{b} \alpha_{b}-\left(1-2 \mu_{b}\right)\left(1-3 \cos ^{2} \theta_{a}\right) \sin \theta_{a} \sin 2 \theta_{b} \alpha_{a}$ \\
\hline
\end{tabular}

Table 10. Same as table 9 , for the $0^{1 / 2} \rightarrow a^{3 / 2} b^{1} \rightarrow 1^{1 / 2} 2^{0} 3^{1 / 2} 4^{1 / 2}$ process in which particle $a$ has spin $3 / 2$ instead of $1 / 2$. This table generalises table 2 . 


\begin{tabular}{|c|c|c|c|}
\hline+3 & $\left(\left|A_{+}\right|^{2}-\left|A_{-}\right|^{2}\right)+\left(\left|A_{+}\right|^{2}+\left|A_{-}\right|^{2}\right) \tilde{P}_{z}$ & & $\cos \theta_{a} \cos ^{2} \theta_{b} \alpha_{a}$ \\
\hline$+3 / 2$ & $\left(\left|B_{+}\right|^{2}-\left|B_{-}\right|^{2}\right)-\left(\left|B_{+}\right|^{2}+\left|B_{-}\right|^{2}\right) \tilde{P}_{z}$ & & $\cos \theta_{a} \sin ^{2} \theta_{b} \alpha_{a}$ \\
\hline+3 & $\left(\left|A_{+}\right|^{2}-\left|A_{-}\right|^{2}\right) \tilde{P}_{z}+\left(\left|A_{+}\right|^{2}+\left|A_{-}\right|^{2}\right)$ & & $\cos ^{2} \theta_{b}$ \\
\hline$-3 / 2$ & $\left(\left|B_{+}\right|^{2}-\left|B_{-}\right|^{2}\right) \tilde{P}_{z}-\left(\left|B_{+}\right|^{2}+\left|B_{-}\right|^{2}\right)$ & & $\sin ^{2} \theta_{b}$ \\
\hline+6 & $\operatorname{Re}\left\{A_{+}^{*} A_{-}\right\} \tilde{P}_{x}-\operatorname{Im}\left\{A_{+}^{*} A_{-}\right\} \tilde{P}_{y}$ & $\cos \phi_{a}$ & $\sin \theta_{a} \cos ^{2} \theta_{b} \alpha_{a}$ \\
\hline$-3 / \sqrt{2}$ & $\left.\operatorname{Re}\left\{A_{+}^{*} B_{+}\right\}-\operatorname{Re}\left\{A_{-}^{*} B_{-}\right\}\right) \tilde{P}_{x}-\left(\operatorname{Im}\left\{A_{+}^{*} B_{+}\right\}+\operatorname{Im}\left\{A_{-}^{*} B_{-}\right\}\right) \tilde{P}_{y}$ & $\cos \phi_{b}$ & $\sin 2 \theta_{b}$ \\
\hline$-3 / \sqrt{2}$ & $\left.\operatorname{Re}\left\{A_{+}^{*} B_{+}\right\}+\operatorname{Re}\left\{A_{-}^{*} B_{-}\right\}\right) \tilde{P}_{x}-\left(\operatorname{Im}\left\{A_{+}^{*} B_{+}\right\}-\operatorname{Im}\left\{A_{-}^{*} B_{-}\right\}\right) \tilde{P}_{y}$ & $\cos \phi_{b}$ & $\cos \theta_{a} \sin 2 \theta_{b} \alpha_{a}$ \\
\hline-3 & $\operatorname{Re}\left\{B_{+}^{*} B_{-}\right\} \tilde{P}_{x}+\operatorname{Im}\left\{B_{+}^{*} B_{-}\right\} \tilde{P}_{y}$ & $\cos \left(\phi_{a}+2 \phi_{b}\right)$ & $\sin \theta_{a} \sin ^{2} \theta_{b} \alpha_{a}$ \\
\hline$+3 / \sqrt{2}$ & $\left(\operatorname{Re}\left\{A_{+}^{*} B_{-}\right\}-\operatorname{Re}\left\{A_{-}^{*} B_{+}\right\}\right)+\left(\operatorname{Re}\left\{A_{+}^{*} B_{-}\right\}+\operatorname{Re}\left\{A_{-}^{*} B_{+}\right\}\right) \tilde{P}_{z}$ & $\cos \left(\phi_{a}+\phi_{b}\right)$ & $\sin \theta_{a} \sin 2 \theta_{b} \alpha_{a}$ \\
\hline+6 & $\operatorname{Re}\left\{A_{+}^{*} A_{-}\right\} \tilde{P}_{y}+\operatorname{Im}\left\{A_{+}^{*} A_{-}\right\} \tilde{P}_{x}$ & $\sin \phi_{a}$ & $\sin \theta_{a} \cos ^{2} \theta_{b} \alpha_{a}$ \\
\hline$+3 / \sqrt{2}$ & $\left.\operatorname{Re}\left\{A_{+}^{*} B_{+}\right\}-\operatorname{Re}\left\{A_{-}^{*} B_{-}\right\}\right) \tilde{P}_{y}+\left(\operatorname{Im}\left\{A_{+}^{*} B_{+}\right\}+\operatorname{Im}\left\{A_{-}^{*} B_{-}\right\}\right) \tilde{P}_{x}$ & $\sin \phi_{b}$ & $\sin 2 \theta_{b}$ \\
\hline$+3 / \sqrt{2}$ & $\left.\operatorname{Re}\left\{A_{+}^{*} B_{+}\right\}+\operatorname{Re}\left\{A_{-}^{*} B_{-}\right\}\right) \tilde{P}_{y}+\left(\operatorname{Im}\left\{A_{+}^{*} B_{+}\right\}-\operatorname{Im}\left\{A_{-}^{*} B_{-}\right\}\right) \tilde{P}_{x}$ & $\sin \phi_{b}$ & $\cos \theta_{a} \sin 2 \theta_{b} \alpha_{a}$ \\
\hline+3 & $\operatorname{Re}\left\{B_{+}^{*} B_{-}\right\} \tilde{P}_{y}-\operatorname{Im}\left\{B_{+}^{*} B_{-}\right\} \tilde{P}_{x}$ & $\sin \left(\phi_{a}+2 \phi_{b}\right)$ & $\sin \theta_{a} \sin ^{2} \theta_{b} \alpha_{a}$ \\
\hline$+3 / \sqrt{2}$ & $\left.\operatorname{Im}\left\{A_{+}^{*} B_{-}\right\}-\operatorname{Im}\left\{A_{-}^{*} B_{+}\right\}\right) \tilde{P}_{z}+\left(\operatorname{Im}\left\{A_{+}^{*} B_{-}\right\}+\operatorname{Im}\left\{A_{-}^{*} B_{+}\right\}\right)$ & $\sin \left(\phi_{a}+\phi_{b}\right)$ & $\sin \theta_{a} \sin 2 \theta_{b} \alpha_{a}$ \\
\hline
\end{tabular}

Table 11. All contributions to $0^{1 / 2} \rightarrow a^{1 / 2} b^{1} \rightarrow 1^{1 / 2} 2^{0} 3^{0} 4^{0}$ angular distribution which appear with the assumptions leading to table 3 are relaxed, so that $P_{x}$ and $P_{y}$ are nonvanishing. We have defined $\left(\tilde{P}_{x}, \tilde{P}_{y}, \tilde{P}_{z}\right) \equiv R(\phi, \theta, 0)^{T}\left(P_{x}, P_{y}, P_{z}\right)$. 


\begin{tabular}{|c|c|c|c|}
\hline \multicolumn{3}{|c|}{$\left(\left|A_{+}\right|^{2}-\left|A_{-}\right|^{2}\right)+\left(\left|A_{+}\right|^{2}+\left|A_{-}\right|^{2}\right) \tilde{P}_{z}$} & $\left(5-9 \cos ^{2} \theta_{a}\right) \cos \theta_{a} \cos ^{2} \theta_{b} \alpha_{a}$ \\
\hline$+3 / 2$ & \multicolumn{2}{|l|}{$\left(\left|A_{+}\right|^{2}-\left|A_{-}\right|^{2}\right) \tilde{P}_{z}+\left(\left|A_{+}\right|^{2}+\left|A_{-}\right|^{2}\right)$} & $\left(1+3 \cos ^{2} \theta_{a}\right) \cos ^{2} \theta_{b}$ \\
\hline$+9 / 4$ & \multicolumn{2}{|l|}{$\left(3\left|B_{+}\right|^{2}-3\left|B_{-}\right|^{2}-\left|C_{+}\right|^{2}+\left|C_{-}\right|^{2}\right)-\left(3\left|B_{+}\right|^{2}+3\left|B_{-}\right|^{2}+\left|C_{+}\right|^{2}+\left|C_{-}\right|^{2}\right) \tilde{P}_{z}$} & $\cos ^{3} \theta_{a} \sin ^{2} \theta_{b} \alpha_{a}$ \\
\hline$-9 / 4$ & \multicolumn{2}{|l|}{$\left(\left|B_{+}\right|^{2}-\left|B_{-}\right|^{2}+\left|C_{+}\right|^{2}-\left|C_{-}\right|^{2}\right) \tilde{P}_{z}-\left(\left|B_{+}\right|^{2}+\left|B_{-}\right|^{2}-\left|C_{+}\right|^{2}-\left|C_{-}\right|^{2}\right)$} & $\cos ^{2} \theta_{a} \sin ^{2} \theta_{b}$ \\
\hline$-3 / 4 \quad($ & \multicolumn{2}{|l|}{$\left(5\left|B_{+}\right|^{2}-5\left|B_{-}\right|^{2}-3\left|C_{+}\right|^{2}+3\left|C_{-}\right|^{2}\right)-\left(5\left|B_{+}\right|^{2}+5\left|B_{-}\right|^{2}+3\left|C_{+}\right|^{2}+3\left|C_{-}\right|^{2}\right) \tilde{P}_{z}$} & $\cos \theta_{a} \sin ^{2} \theta_{b} \alpha_{a}$ \\
\hline$-3 / 4$ & \multicolumn{2}{|l|}{$\left(\left|B_{+}\right|^{2}-\left|B_{-}\right|^{2}-3\left|C_{+}\right|^{2}+3\left|C_{-}\right|^{2}\right) \tilde{P}_{z}-\left(\left|B_{+}\right|^{2}+\left|B_{-}\right|^{2}+3\left|C_{+}\right|^{2}+3\left|C_{-}\right|^{2}\right)$} & $\sin ^{2} \theta_{b}$ \\
\hline$+3 \sqrt{3} / 2$ & $\left(\operatorname{Re}\left\{B_{+}^{*} C_{+}\right\}+\operatorname{Re}\left\{B_{-}^{*} C_{-}\right\}\right) \tilde{P}_{x}+\left(\operatorname{Im}\left\{B_{+}^{*} C_{+}\right\}-\operatorname{Im}\left\{B_{-}^{*} C_{-}\right\}\right) \tilde{P}_{y}$ & $\cos \phi_{a}$ & $\left(1-3 \cos ^{2} \theta_{a}\right) \sin \theta_{a} \sin ^{2} \theta_{b} \alpha_{a}$ \\
\hline-3 & $\operatorname{Re}\left\{A_{+}^{*} A_{-}\right\} \tilde{P}_{x}-\operatorname{Im}\left\{A_{+}^{*} A_{-}\right\} \tilde{P}_{y}$ & $\cos \phi_{a}$ & $\left(1-9 \cos ^{2} \theta_{a}\right) \sin \theta_{a} \cos ^{2} \theta_{b} \alpha_{a}$ \\
\hline$-3 \sqrt{3} / 2$ & $\left(\operatorname{Re}\left\{B_{+}^{*} C_{+}\right\}-\operatorname{Re}\left\{B_{-}^{*} C_{-}\right\}\right) \tilde{P}_{x}+\left(\operatorname{Im}\left\{B_{+}^{*} C_{+}\right\}+\operatorname{Im}\left\{B_{-}^{*} C_{-}\right\}\right) \tilde{P}_{y}$ & $\cos \phi_{a}$ & $\sin 2 \theta_{a} \sin ^{2} \theta_{b}$ \\
\hline$+3 / 2 \sqrt{2}$ & $\left(\operatorname{Re}\left\{A_{+}^{*} B_{+}\right\}+\operatorname{Re}\left\{A_{-}^{*} B_{-}\right\}\right) \tilde{P}_{x}-\left(\operatorname{Im}\left\{A_{+}^{*} B_{+}\right\}-\operatorname{Im}\left\{A_{-}^{*} B_{-}\right\}\right) \tilde{P}_{y}$ & $\cos \phi_{b}$ & $\left(5-9 \cos ^{2} \theta_{a}\right) \cos \theta_{a} \sin 2 \theta_{b} \alpha_{a}$ \\
\hline$-3 / 2 \sqrt{2}$ & $\left(\operatorname{Re}\left\{A_{+}^{*} B_{+}\right\}-\operatorname{Re}\left\{A_{-}^{*} B_{-}\right\}\right) \tilde{P}_{x}-\left(\operatorname{Im}\left\{A_{+}^{*} B_{+}\right\}+\operatorname{Im}\left\{A_{-}^{*} B_{-}\right\}\right) \tilde{P}_{y}$ & $\phi_{b}$ & $\left(1+3 \cos ^{2} \theta_{a}\right) \sin 2 \theta_{b}$ \\
\hline$+9 / 2$ & $\operatorname{Re}\left\{C_{+}^{*} C_{-}\right\} \tilde{P}_{x}-\operatorname{Im}\left\{C_{+}^{*} C_{-}\right\} \tilde{P}_{y}$ & $\cos \left(3 \phi_{a}+2 \phi_{b}\right)$ & $\sin \theta_{a}^{3} \sin ^{2} \theta_{b} \alpha_{a}$ \\
\hline$-9 \sqrt{3} / 2$ & $\left(\operatorname{Re}\left\{B_{+}^{*} C_{-}\right\}-\operatorname{Re}\left\{B_{-}^{*} C_{+}\right\}\right)-\left(\operatorname{Re}\left\{B_{+}^{*} C_{-}\right\}+\operatorname{Re}\left\{B_{-}^{*} C_{+}\right\}\right) \tilde{P}_{z}$ & $\cos \left(2 \phi_{a}+2 \phi_{b}\right)$ & $\sin \theta_{a}^{2} \cos \theta_{a} \sin ^{2} \theta_{b} \alpha_{a}$ \\
\hline$-3 \sqrt{3} / 2$ & $\left(\operatorname{Re}\left\{B_{+}^{*} C_{-}\right\}-\operatorname{Re}\left\{B_{-}^{*} C_{+}\right\}\right) \tilde{P}_{z}-\left(\operatorname{Re}\left\{B_{+}^{*} C_{-}\right\}+\operatorname{Re}\left\{B_{-}^{*} C_{+}\right\}\right)$ & $\cos \left(2 \phi_{a}+2 \phi_{b}\right)$ & $\sin \theta_{a}^{2} \sin ^{2} \theta_{b}$ \\
\hline$+9 \sqrt{2 / 3} / 2$ & $\left(\operatorname{Re}\left\{A_{+}^{*} C_{-}\right\}+\operatorname{Re}\left\{A_{-}^{*} C_{+}\right\}\right) \tilde{P}_{x}-\left(\operatorname{Im}\left\{A_{+}^{*} C_{-}\right\}-\operatorname{Im}\left\{A_{-}^{*} C_{+}\right\}\right) \tilde{P}_{y}$ & $\cos \left(2 \phi_{a}+\phi_{b}\right)$ & $\sin \theta_{a}^{2} \cos \theta_{a} \sin 2 \theta_{b} \alpha_{a}$ \\
\hline$-3 \sqrt{2 / 3} / 2$ & $\left(\operatorname{Re}\left\{A_{+}^{*} C_{-}\right\}-\operatorname{Re}\left\{A_{-}^{*} C_{+}\right\}\right) \tilde{P}_{x}-\left(\operatorname{Im}\left\{A_{+}^{*} C_{-}\right\}+\operatorname{Im}\left\{A_{-}^{*} C_{+}\right\}\right) \tilde{P}_{y}$ & $\cos \left(2 \phi_{a}+\phi_{b}\right)$ & $\sin \theta_{a}^{2} \sin 2 \theta_{b}$ \\
\hline$+3 / 2$ & $\operatorname{Re}\left\{B_{+}^{*} B_{-}\right\} \tilde{P}_{x}+\operatorname{Im}\left\{B_{+}^{*} B_{-}\right\} \tilde{P}_{y}$ & $\cos \left(\phi_{a}+2 \phi_{b}\right)$ & $\left(1-9 \cos ^{2} \theta_{a}\right) \sin \theta_{a} \sin ^{2} \theta_{b} \alpha_{a}$ \\
\hline$-3 \sqrt{2 / 3} / 2$ & $\left(\operatorname{Re}\left\{A_{+}^{*} C_{+}\right\}-\operatorname{Re}\left\{A_{-}^{*} C_{-}\right\}\right)+\left(\operatorname{Re}\left\{A_{+}^{*} C_{+}\right\}+\operatorname{Re}\left\{A_{-}^{*} C_{-}\right\}\right) \tilde{P}_{z}$ & $\cos \left(\phi_{a}+\phi_{b}\right)$ & $\left(1-3 \cos ^{2} \theta_{a}\right) \sin \theta_{a} \sin 2 \theta_{b} \alpha_{a}$ \\
\hline$-3 / 2 \sqrt{2}$ & $\left(\operatorname{Re}\left\{A_{+}^{*} B_{-}\right\}-\operatorname{Re}\left\{A_{-}^{*} B_{+}\right\}\right)+\left(\operatorname{Re}\left\{A_{+}^{*} B_{-}\right\}+\operatorname{Re}\left\{A_{-}^{*} B_{+}\right\}\right) \tilde{P}_{z}$ & $\cos \left(\phi_{a}+\phi_{b}\right)$ & $\left(1-9 \cos ^{2} \theta_{a}\right) \sin \theta_{a} \sin 2 \theta_{b} \alpha_{a}$ \\
\hline$+3 \sqrt{2 / 3} / 2$ & $\left(\operatorname{Re}\left\{A_{+}^{*} C_{+}\right\}-\operatorname{Re}\left\{A_{-}^{*} C_{-}\right\}\right) \tilde{P}_{z}+\left(\operatorname{Re}\left\{A_{+}^{*} C_{+}\right\}+\operatorname{Re}\left\{A_{-}^{*} C_{-}\right\}\right)$ & $\cos \left(\phi_{a}+\phi_{b}\right)$ & $\sin 2 \theta_{a} \sin 2 \theta_{b}$ \\
\hline$+3 \sqrt{3} / 2$ & $\left(\operatorname{Re}\left\{B_{+}^{*} C_{+}\right\}+\operatorname{Re}\left\{B_{-}^{*} C_{-}\right\}\right) \tilde{P}_{y}-\left(\operatorname{Im}\left\{B_{+}^{*} C_{+}\right\}-\operatorname{Im}\left\{B_{-}^{*} C_{-}\right\}\right) \tilde{P}_{x}$ & $\sin \phi_{a}$ & $\left(1-3 \cos ^{2} \theta_{a}\right) \sin \theta_{a} \sin ^{2} \theta_{b} \alpha_{a}$ \\
\hline-3 & $\operatorname{Re}\left\{A_{+}^{*} A_{-}\right\} \tilde{P}_{y}+\operatorname{Im}\left\{A_{+}^{*} A_{-}\right\} \tilde{P}_{x}$ & $\sin \phi_{a}$ & $\left(1-9 \cos ^{2} \theta_{a}\right) \sin \theta_{a} \cos ^{2} \theta_{b} \alpha_{a}$ \\
\hline$-3 \sqrt{3} / 2$ & $\left(\operatorname{Re}\left\{B_{+}^{*} C_{+}\right\}-\operatorname{Re}\left\{B_{-}^{*} C_{-}\right\}\right) \tilde{P}_{y}-\left(\operatorname{Im}\left\{B_{+}^{*} C_{+}\right\}+\operatorname{Im}\left\{B_{-}^{*} C_{-}\right\}\right) \tilde{P}_{x}$ & $\sin \phi_{a}$ & $\sin 2 \theta_{a} \sin ^{2} \theta_{b}$ \\
\hline$-3 / 2 \sqrt{2}$ & $\left(\operatorname{Re}\left\{A_{+}^{*} B_{+}\right\}+\operatorname{Re}\left\{A_{-}^{*} B_{-}\right\}\right) \tilde{P}_{y}+\left(\operatorname{Im}\left\{A_{+}^{*} B_{+}\right\}-\operatorname{Im}\left\{A_{-}^{*} B_{-}\right\}\right) \tilde{P}_{x}$ & $\sin \phi_{b}$ & $\left(5-9 \cos ^{2} \theta_{a}\right) \cos \theta_{a} \sin 2 \theta_{b} \alpha_{a}$ \\
\hline$+3 / 2 \sqrt{2}$ & $\left(\operatorname{Re}\left\{A_{+}^{*} B_{+}\right\}-\operatorname{Re}\left\{A_{-}^{*} B_{-}\right\}\right) \tilde{P}_{y}+\left(\operatorname{Im}\left\{A_{+}^{*} B_{+}\right\}+\operatorname{Im}\left\{A_{-}^{*} B_{-}\right\}\right) \tilde{P}_{x}$ & $\sin \phi_{b}$ & $\left(1+3 \cos ^{2} \theta_{a}\right) \sin 2 \theta_{b}$ \\
\hline$+9 / 2$ & $\operatorname{Re}\left\{C_{+}^{*} C_{-}\right\} \tilde{P}_{y}+\operatorname{Im}\left\{C_{+}^{*} C_{-}\right\} \tilde{P}_{x}$ & $\sin \left(3 \phi_{a}+2 \phi_{b}\right)$ & $\sin \theta_{a}^{3} \sin ^{2} \theta_{b} \alpha_{a}$ \\
\hline$+9 \sqrt{3} / 2$ & $\left(\operatorname{Im}\left\{B_{+}^{*} C_{-}\right\}-\operatorname{Im}\left\{B_{-}^{*} C_{+}\right\}\right) \tilde{P}_{z}-\left(\operatorname{Im}\left\{B_{+}^{*} C_{-}\right\}+\operatorname{Im}\left\{B_{-}^{*} C_{+}\right\}\right)$ & $\sin \left(2 \phi_{a}+2 \phi_{b}\right)$ & $\sin \theta_{a}^{2} \cos \theta_{a} \sin ^{2} \theta_{b} \alpha_{a}$ \\
\hline$+3 \sqrt{3} / 2$ & $\left(\operatorname{Im}\left\{B_{+}^{*} C_{-}\right\}-\operatorname{Im}\left\{B_{-}^{*} C_{+}\right\}\right)-\left(\operatorname{Im}\left\{B_{+}^{*} C_{-}\right\}+\operatorname{Im}\left\{B_{-}^{*} C_{+}\right\}\right) \tilde{P}_{z}$ & $\sin \left(2 \phi_{a}+2 \phi_{b}\right)$ & $\sin \theta_{a}^{2} \sin ^{2} \theta_{b}$ \\
\hline$+9 \sqrt{2 / 3} / 2$ & $\left(\operatorname{Re}\left\{A_{+}^{*} C_{-}\right\}+\operatorname{Re}\left\{A_{-}^{*} C_{+}\right\}\right) \tilde{P}_{y}+\left(\operatorname{Im}\left\{A_{+}^{*} C_{-}\right\}-\operatorname{Im}\left\{A_{-}^{*} C_{+}\right\}\right) \tilde{P}_{x}$ & $\sin \left(2 \phi_{a}+\phi_{b}\right)$ & $\sin \theta_{a}^{2} \cos \theta_{a} \sin 2 \theta_{b} \alpha_{a}$ \\
\hline$-3 \sqrt{2 / 3} / 2$ & $\left(\operatorname{Re}\left\{A_{+}^{*} C_{-}\right\}-\operatorname{Re}\left\{A_{-}^{*} C_{+}\right\}\right) \tilde{P}_{y}+\left(\operatorname{Im}\left\{A_{+}^{*} C_{-}\right\}+\operatorname{Im}\left\{A_{-}^{*} C_{+}\right\}\right) \tilde{P}_{x}$ & $\sin \left(2 \phi_{a}+\phi_{b}\right)$ & $\sin \theta_{a}^{2} \sin 2 \theta_{b}$ \\
\hline$-3 / 2$ & $\operatorname{Re}\left\{B_{+}^{*} B_{-}\right\} \tilde{P}_{y}-\operatorname{Im}\left\{B_{+}^{*} B_{-}\right\} \tilde{P}_{x}$ & $\sin \left(\phi_{a}+2 \phi_{b}\right)$ & $\left(1-9 \cos ^{2} \theta_{a}\right) \sin \theta_{a} \sin ^{2} \theta_{b} \alpha_{a}$ \\
\hline$+3 \sqrt{2 / 3} / 2$ & $\left(\operatorname{Im}\left\{A_{+}^{*} C_{+}\right\}-\operatorname{Im}\left\{A_{-}^{*} C_{-}\right\}\right) \tilde{P}_{z}+\left(\operatorname{Im}\left\{A_{+}^{*} C_{+}\right\}+\operatorname{Im}\left\{A_{-}^{*} C_{-}\right\}\right)$ & $\sin \left(\phi_{a}+\phi_{b}\right)$ & $\left(1-3 \cos ^{2} \theta_{a}\right) \sin \theta_{a} \sin 2 \theta_{b} \alpha_{a}$ \\
\hline$-3 / 2 \sqrt{2}$ & $\left(\operatorname{Im}\left\{A_{+}^{*} B_{-}\right\}-\operatorname{Im}\left\{A_{-}^{*} B_{+}\right\}\right) \tilde{P}_{z}+\left(\operatorname{Im}\left\{A_{+}^{*} B_{-}\right\}+\operatorname{Im}\left\{A_{-}^{*} B_{+}\right\}\right)$ & $\sin \left(\phi_{a}+\phi_{b}\right)$ & $\left(1-9 \cos ^{2} \theta_{a}\right) \sin \theta_{a} \sin 2 \theta_{b} \alpha_{a}$ \\
\hline$-3 \sqrt{2 / 3} / 2$ & $\left(\operatorname{Im}\left\{A_{+}^{*} C_{+}\right\}-\operatorname{Im}\left\{A_{-}^{*} C_{-}\right\}\right)+\left(\operatorname{Im}\left\{A_{+}^{*} C_{+}\right\}+\operatorname{Im}\left\{A_{-}^{*} C_{-}\right\}\right) \tilde{P}_{z}$ & $\sin \left(\phi_{a}+\phi_{b}\right)$ & $\sin 2 \theta_{a} \sin 2 \theta_{b}$ \\
\hline
\end{tabular}

Table 12. Same as table 11 , for the $0^{1 / 2} \rightarrow a^{3 / 2} b^{1} \rightarrow 1^{1 / 2} 2^{0} 3^{0} 4^{0}$ process, in which particle $a$ has spin $3 / 2$ instead of $1 / 2$. This table generalises table 4 . 


$$
\begin{aligned}
& +\left(\left|A_{+}\right|^{2}-\left|A_{-}\right|^{2}-\left|B_{+}\right|^{2}+\left|B_{-}\right|^{2}\right) \tilde{P}_{z}+\left(\left|A_{+}\right|^{2}+\left|A_{-}\right|^{2}+\left|B_{+}\right|^{2}+\left|B_{-}\right|^{2}\right) \\
& +\left(\left|A_{+}\right|^{2}-\left|A_{-}\right|^{2}+\left|B_{+}\right|^{2}-\left|B_{-}\right|^{2}\right)+\left(\left|A_{+}\right|^{2}+\left|A_{-}\right|^{2}-\left|B_{+}\right|^{2}-\left|B_{-}\right|^{2}\right) \tilde{P}_{z} \\
& +2 \quad \operatorname{Re}\left\{A_{+}^{*} A_{-}\right\} \tilde{P}_{x}-\operatorname{Im}\left\{A_{+}^{*} A_{-}\right\} \tilde{P}_{y} \\
& +2 \quad \operatorname{Re}\left\{A_{+}^{*} A_{-}\right\} \tilde{P}_{y}+\operatorname{Im}\left\{A_{+}^{*} A_{-}\right\} \tilde{P}_{x}
\end{aligned}
$$

Table 13. All contributions to $0^{1 / 2} \rightarrow a^{1 / 2} b^{1} \rightarrow 1^{1 / 2} 2^{0} b^{1}$ angular distribution which appear when the assumptions leading to table 5 are relaxed, so that $A_{ \pm}, P_{x}, P_{y}$ defined in the text are nonvanishing.

\begin{tabular}{|cccc}
\hline$+1 / 2$ & $\left(\left|A_{+}\right|^{2}-\left|A_{-}\right|^{2}-\left|B_{+}\right|^{2}+\left|B_{-}\right|^{2}+3\left|C_{+}\right|^{2}-3\left|C_{-}\right|^{2}\right) \tilde{P}_{z}+\left(\left|A_{+}\right|^{2}+\left|A_{-}\right|^{2}+\left|B_{+}\right|^{2}+\left|B_{-}\right|^{2}+3\left|C_{+}\right|^{2}+3\left|C_{-}\right|^{2}\right)$ & \\
$+3 / 2$ & $\left(3\left|A_{+}\right|^{2}-3\left|A_{-}\right|^{2}+3\left|B_{+}\right|^{2}-3\left|B_{-}\right|^{2}-\left|C_{+}\right|^{2}+\left|C_{-}\right|^{2}\right)+\left(3\left|A_{+}\right|^{2}+3\left|A_{-}\right|^{2}-3\left|B_{+}\right|^{2}-3\left|B_{-}\right|^{2}-\left|C_{+}\right|^{2}-\left|C_{-}\right|^{2}\right) \tilde{P}_{z}$ & $\cos ^{3} \theta_{a} \alpha_{a}$ \\
$+3 / 2$ & $\left(\left|A_{+}\right|^{2}-\left|A_{-}\right|^{2}-\left|B_{+}\right|^{2}+\left|B_{-}\right|^{2}-\left|C_{+}\right|^{2}+\left|C_{-}\right|^{2}\right) \tilde{P}_{z}+\left(\left|A_{+}\right|^{2}+\left|A_{-}\right|^{2}+\left|B_{+}\right|^{2}+\left|B_{-}\right|^{2}-\left|C_{+}\right|^{2}-\left|C_{-}\right|^{2}\right)$ & $\cos ^{2} \theta_{a}$ \\
$-1 / 2$ & $\left(5\left|A_{+}\right|^{2}-5\left|A_{-}\right|^{2}+5\left|B_{+}\right|^{2}-5\left|B_{-}\right|^{2}-3\left|C_{+}\right|^{2}+3\left|C_{-}\right|^{2}\right)+\left(5\left|A_{+}\right|^{2}+5\left|A_{-}\right|^{2}-5\left|B_{+}\right|^{2}-5\left|B_{-}\right|^{2}-3\left|C_{+}\right|^{2}-3\left|C_{-}\right|^{2}\right) \tilde{P}_{z}$ & $\cos _{a} \alpha_{a}$ \\
$+\sqrt{3}$ & $\left(\operatorname{Re}\left\{B_{+}^{*} C_{+}\right\}+\operatorname{Re}\left\{B_{-}^{*} C_{-}\right\}\right) \tilde{P}_{x}+\left(\operatorname{Im}\left\{B_{+}^{*} C_{+}\right\}-\operatorname{Im}\left\{B_{-}^{*} C_{-}\right\}\right) \tilde{P}_{y}$ & $\cos \phi_{a}$ & $\left(1-3 \cos ^{2} \theta_{a}\right) \sin \theta_{a} \alpha_{a}$ \\
- & $\operatorname{Re}\left\{A_{+}^{*} A_{-}\right\} \tilde{P}_{x}-\operatorname{Im}\left\{A_{+}^{*} A_{-}\right\} \tilde{P}_{y}$ & $\cos \phi_{a}$ & $\left(1-9 \cos \theta_{a}\right) \sin \theta_{a} \alpha_{a}$ \\
$-2 \sqrt{3}$ & $\left(\operatorname{Re}\left\{B_{+}^{*} C_{+}\right\}-\operatorname{Re}\left\{B_{-}^{*} C_{-}\right\}\right) \tilde{P}_{x}+\left(\operatorname{Im}\left\{B_{+}^{*} C_{+}\right\}+\operatorname{Im}\left\{B_{-}^{*} C_{-}\right\}\right) \tilde{P}_{y}$ & $\cos \phi_{a}$ & $\cos \theta_{a} \sin \theta_{a}$ \\
$+\sqrt{3}$ & $\left(\operatorname{Re}\left\{B_{+}^{*} C_{+}\right\}+\operatorname{Re}\left\{B_{-}^{*} C_{-}\right\}\right) \tilde{P}_{y}-\left(\operatorname{Im}\left\{B_{+}^{*} C_{+}\right\}-\operatorname{Im}\left\{B_{-}^{*} C_{-}\right\}\right) \tilde{P}_{x}$ & $\sin \phi_{a}$ & $\left(1-3 \cos \theta_{a}\right) \sin \theta_{a} \alpha_{a}$ \\
- & $\operatorname{Re}\left\{A_{+}^{*} A_{-}\right\} \tilde{P}_{y}+\operatorname{Im}\left\{A_{+}^{*} A_{-}\right\} \tilde{P}_{x}$ & $\sin \phi_{a}$ & $\left.\left(1-9 \cos ^{2} \theta_{a}\right) \sin \theta_{a} \alpha_{a}\right\}$ \\
$-2 \sqrt{3}$ & $\left(\operatorname{Re}\left\{B_{+}^{*} C_{+}\right\}-\operatorname{Re}\left\{B_{-}^{*} C_{-}\right\}\right) \tilde{P}_{y}-\left(\operatorname{Im}\left\{B_{+}^{*} C_{+}\right\}+\operatorname{Im}\left\{B_{-}^{*} C_{-}\right\}\right) \tilde{P}_{x}$ & $\sin \phi_{a}$ & $\cos \theta_{a} \sin \theta_{a}$ \\
\hline
\end{tabular}

Table 14. Same as table 13, for the $0^{1 / 2} \rightarrow a^{3 / 2} b^{1} \rightarrow 1^{1 / 2} 2^{0} b^{1}$ process, in which particle $a$ has spin $3 / 2$ instead of $1 / 2$. This table generalises table 6 .

$$
\begin{array}{|cccc}
+ & \left(\left|A_{+}\right|^{2}-\left|A_{-}\right|^{2}\right) \tilde{P}_{z}+\left(\left|A_{+}\right|^{2}+\left|A_{-}\right|^{2}\right) & \\
+ & \left(\left|A_{+}\right|^{2}-\left|A_{-}\right|^{2}\right)+\left(\left|A_{+}\right|^{2}+\left|A_{-}\right|^{2}\right) \tilde{P}_{z} & \cos \theta_{a} \alpha_{a} \\
+2 & \operatorname{Re}\left\{A_{+}^{*} A_{-}\right\} \tilde{P}_{x}-\operatorname{Im}\left\{A_{+}^{*} A_{-}\right\} \tilde{P}_{y} & \cos \phi_{a} & \sin \theta_{a} \alpha_{a} \\
+2 & \operatorname{Re}\left\{A_{+}^{*} A_{-}\right\} \tilde{P}_{y}+\operatorname{Im}\left\{A_{+}^{*} A_{-}\right\} \tilde{P}_{x} & \sin \phi_{a} & \sin \theta_{a} \alpha_{a}
\end{array}
$$

Table 15. All contributions to $0^{1 / 2} \rightarrow a^{1 / 2} b^{0} \rightarrow 1^{1 / 2} 2^{0} b^{0}$ angular distribution which appear when the assumptions leading to table 7 are relaxed, so that $P_{x}, P_{y}$ defined in the text are nonvanishing.

$$
\begin{aligned}
& -1 / 2 \quad\left(\left|A_{+}\right|^{2}-\left|A_{-}\right|^{2}\right)+\left(\left|A_{+}\right|^{2}+\left|A_{-}\right|^{2}\right) \tilde{P}_{z} \quad\left(5-9 \cos ^{2} \theta_{a}\right) \cos \theta_{a} \alpha_{a} \\
& +1 / 2 \quad\left(\left|A_{+}\right|^{2}-\left|A_{-}\right|^{2}\right) \tilde{P}_{z}+\left(\left|A_{+}\right|^{2}+\left|A_{-}\right|^{2}\right) \quad\left(1+3 \cos ^{2} \theta_{a}\right) \\
& -\operatorname{Re}\left\{A_{+}^{*} A_{-}\right\} \tilde{P}_{x}-\operatorname{Im}\left\{A_{+}^{*} A_{-}\right\} \tilde{P}_{y} \quad \cos \phi_{a} \quad\left(1-9 \cos ^{2} \theta_{a}\right) \sin \theta_{a} \alpha_{a} \\
& -\operatorname{Re}\left\{A_{+}^{*} A_{-}\right\} \tilde{P}_{y}+\operatorname{Im}\left\{A_{+}^{*} A_{-}\right\} \tilde{P}_{x} \quad \sin \phi_{a} \quad\left(1-9 \cos ^{2} \theta_{a}\right) \sin \theta_{a} \alpha_{a}
\end{aligned}
$$

Table 16. Same as table 15 for the $0^{1 / 2} \rightarrow a^{3 / 2} b^{0} \rightarrow 1^{1 / 2} 2^{0} b^{0}$ process, in which particle $a$ has spin $3 / 2$ instead of $1 / 2$. This table generalises table 8 . 
Open Access. This article is distributed under the terms of the Creative Commons Attribution License (CC-BY 4.0), which permits any use, distribution and reproduction in any medium, provided the original author(s) and source are credited.

\section{References}

[1] M. Vieites Díaz, Charmless two-body b-meson decays at LHCb, presentation at the ICHEP conference, Chicago U.S.A. August 42016.

[2] G. Durieux and Y. Grossman, Probing CP-violation systematically in differential distributions, Phys. Rev. D 92 (2015) 076013 [arXiv:1508.03054] [INSPIRE].

[3] F. Beaujean, M. Chrzaszcz, N. Serra and D. van Dyk, Extracting angular observables without a likelihood and applications to rare decays, Phys. Rev. D 91 (2015) 114012 [arXiv: 1503.04100] [INSPIRE].

[4] J.D. Jackson, Particle and polarization angular distribution for two and three-body decays, in Lectures delivered at the Les Houches summer school of theoretical physics, C. DeWitt and M. Jacob eds., Gordon and Breach, New York U.S.A. (1965), pg. 325 [INSPIRE].

[5] LHCb collaboration, Measurements of the $\Lambda_{b}^{0} \rightarrow J / \psi \Lambda$ decay amplitudes and the $\Lambda_{b}^{0}$ polarisation in pp collisions at $\sqrt{s}=7 \mathrm{TeV}$, Phys. Lett. B 724 (2013) 27 [arXiv:1302.5578] [INSPIRE].

[6] LHCb collaboration, Observation of the $\Lambda_{b}^{0} \rightarrow \Lambda \phi$ decay, Phys. Lett. B 759 (2016) 282 [arXiv: 1603.02870] [INSPIRE].

[7] LHCb collaboration, Searches for $\Lambda_{b}^{0}$ and $\Xi_{b}^{0}$ decays to $K_{\mathrm{S}}^{0} p \pi^{-}$and $K_{\mathrm{S}}^{0} p K^{-}$final states with first observation of the $\Lambda_{b}^{0} \rightarrow K_{\mathrm{S}}^{0} p \pi^{-}$decay, JHEP 04 (2014) 087 [arXiv:1402.0770] [INSPIRE].

[8] M. Gremm, F. Krüger and L.M. Sehgal, Angular distribution and polarization of photons in the inclusive decay $\Lambda_{b} \rightarrow X_{s} \gamma$, Phys. Lett. B 355 (1995) 579 [hep-ph/9505354] [INSPIRE].

[9] T. Mannel and S. Recksiegel, Flavor changing neutral current decays of heavy baryons: the case $\Lambda_{b} \rightarrow \Lambda \gamma$, J. Phys. G 24 (1998) 979 [hep-ph/9701399] [INSPIRE].

[10] G. Hiller and A. Kagan, Probing for new physics in polarized $\Lambda_{b}$ decays at the $Z$, Phys. Rev. D 65 (2002) 074038 [hep-ph/0108074] [INSPIRE].

[11] F. Legger and T. Schietinger, Photon helicity in $\Lambda_{b} \rightarrow p K \gamma$ decays, Phys. Lett. B 645 (2007) 204 [Erratum ibid. B 647 (2007) 527] [hep-ph/0605245] [INSPIRE].

[12] G. Hiller, M. Knecht, F. Legger and T. Schietinger, Photon polarization from helicity suppression in radiative decays of polarized $\Lambda_{b}$ to spin-3/2 baryons, Phys. Lett. B 649 (2007) 152 [hep-ph/0702191] [INSPIRE].

[13] W. Bensalem, A. Datta and D. London, T violating triple product correlations in charmless $\Lambda_{b}$ decays, Phys. Lett. B 538 (2002) 309 [hep-ph/0205009] [INSPIRE].

[14] W. Bensalem, A. Datta and D. London, New physics effects on triple product correlations in $\Lambda_{b}$ decays, Phys. Rev. D 66 (2002) 094004 [hep-ph/0208054] [INSPIRE].

[15] M. Jacob and G.C. Wick, On the general theory of collisions for particles with spin, Annals Phys. 7 (1959) 404 [INSPIRE]. 
[16] E. Conte, Recherche de la violation des symétries $C P$ et $T$ dans les réactions $\Lambda_{b}^{0} \rightarrow \Lambda^{0}+$ un méson vecteur (in French), Ph.D. thesis, CERN-THESIS-2008-027, Clermont-Ferrand U., Clermont-Ferrand France (2008) [INSPIRE].

[17] O. Leitner, Z.J. Ajaltouni and E. Conte, Testing fundamental symmetries with $\Lambda_{b} \rightarrow \Lambda$-vector decay, hep-ph/0602043 [INSPIRE].

[18] O. Leitner and Z.J. Ajaltouni, Testing CP and time reversal symmetries with $\Lambda_{b} \rightarrow \Lambda V(1-)$ decays, Nucl. Phys. Proc. Suppl. 174 (2007) 169 [hep-ph/0610189] [InSPIRE].

[19] J. Hrivnac, R. Lednicky and M. Smizanska, Feasibility of beauty baryon polarization measurement in $\Lambda^{0} J / \psi$ decay channel by ATLAS LHC, J. Phys. G 21 (1995) 629 [hep-ph/9405231] [INSPIRE].

[20] X.-W. Kang, H.-B. Li, G.-R. Lu and A. Datta, Study of CP-violation in $\Lambda_{c}^{+}$decay, Int. J. Mod. Phys. A 26 (2011) 2523 [arXiv: 1003.5494] [InSPIRE].

[21] M. Gronau and J.L. Rosner, Triple product asymmmetries in $\Lambda_{b}$ and $\Xi_{b}$ decays, Phys. Lett. B 749 (2015) 104 [arXiv: 1506.01346] [INSPIRE]. 